\title{
Anglo-Saxon Diet in the Conversion Period: a comparative isotopic study using carbon and nitrogen
}

Emma L. Hannah ${ }^{\text {a, }}{ }^{\text {* }}$, T. Rowan McLaughlin ${ }^{\text {b }}$, and Evelyn M. Keaveney ${ }^{\mathrm{b}}$, and Susanne E. Hakenbeck $^{\text {a }}$

a Department of Archaeology, University of Cambridge, Cambridge CB2 3ER, UK

${ }^{\mathrm{b}}$ School of Natural and Built Environment, Queen's University Belfast, BT7 1NN, UK

* Corresponding author -- ehannah01@qub.ac.uk

Conflicts of interest: none.

\begin{abstract}
Seventh-century Anglo-Saxon England is characterised by great social and religious change.

The arrival of missionaries from Rome in AD 597 initiated the gradual process of conversion to Christianity. There is growing evidence for increasing hierarchy and social stratification in the archaeological record at this time, including prominent kingly burials. This paper investigates whether diet was influenced by social stratification and to a lesser extent religion in two seventh-century cemetery populations: Melbourn, Cambridgeshire, and Polhill, Kent. Analysis of carbon and nitrogen stable isotopes from 116 human individuals was undertaken. Factors considered included age, sex, wealth and other notable grave features. Results showed that the diets of both populations were largely unaffected by these wider social processes, with negligible differences between social groups. The results were placed in the context of wider Anglo-Saxon dietary studies and highlight that Anglo-Saxon populations consistently display overwhelmingly similar ranges of carbon and nitrogen isotopes.
\end{abstract}

Keywords: Anglo-Saxon; Carbon; Nitrogen; Stable isotope analysis; Palaeodiet

\section{Highlights}

- Dietary patterns of two seventh-century Anglo-Saxon populations are examined.

- Little dietary difference found between various social factors.

- Anglo-Saxon cemetery populations display similar isotopic ranges. 


\section{Introduction}

Religious transformation in a society can bring about many changes in human behaviour. Past episodes of such change often gave rise to cultural trajectories that were significantly different from what came before, leading to fundamental shifts in economy, politics, and social stratification; hence an enduring interest for historians and archaeologists. One such episode was England's conversion to Christianity during the Anglo-Saxon period, beginning late in the Sixth Century AD. Copious historical and archaeological data attest to this time being the setting of major social and religious change, including the establishment of a new political structure. Less is known about how these changes affected the rural populace, especially at the fundamentally important level of subsistence economy, but equally unclear is the degree to which social stratification was reflected in diet. Nor is it understood how widely religious conventions with regard to food were adopted beyond the ecclesiastical context in which they were first developed. Palaeodietary studies, specifically stable isotope analyses of skeletal remains, can address these issues, providing direct evidence of the type of food people ate in the past, complimenting what is known from historical records and other forms of archaeological evidence. A diet rich in meat, for example, which would be expected among individuals of high social rank (e.g. Knipper et al. 2015), elevates the $\delta^{15} \mathrm{~N}$ values of bone to a measurable degree (Schoeninger and DeNiro 1984). This paper compares measurements obtained from individuals of differing age, sex and social rank, and thereby investigates the influence of social change on diet.

We analysed stable carbon and nitrogen isotopes in samples of bone collagen from two sixth- to eighth-century Anglo-Saxon cemeteries: Polhill, Kent, and Melbourn, Cambridgeshire. We investigated whether wider social and religious changes of the period had an impact on everyday diet, as well as how the cemeteries compared to Anglo-Saxon dietary studies published elsewhere. We reconstructed dietary patterns to compare and 
understand ways in which communities imposed social stratification, using age, sex and wealth as parameters in the study. The presence of various grave forms at Polhill, and body positions at Melbourn (archaeological indicators of stratification), provided another factor by which to investigate whether distinct burial differences were related to diet.

\subsection{Historical context}

The cemeteries were in use during a transformative period. Over the course of the sixth and seventh centuries, England's conversion to Christianity began in AD 597, when St Augustine began his mission in the kingdom of Kent (Blair 2005; Higham \& Ryan 2013). Conversion occurred at different times throughout England, marking a period of transition (Hoggett 2010). Pagan and Christian religious and burial practices were mixed, as exemplified by the ship burial at Sutton Hoo (Carver 1998). Pertinent to our study, the fifthcentury Rule of St Benedict, a book of instructions written by the saint for religious persons in communal living within monasteries, outlines fasting and food stipulations imposed on monastic communities and Christians for a substantial portion of the year (Simon 2009). It is not known when exactly these restrictions were enforced among the lay population. Banham (2004) discusses the adherence of such regulations by Anglo-Saxon monastic communities and their reliance on fish for this purpose. Fish is commonly thought to have been the foodstuff consumed during fasting (Simoons 1994) but, as Frantzen (2014) points out, little written evidence exists from the period which details the diet of lay people or links fish consumption to religion. Fish consumption linked to Christian fasting practices has been revealed in isotope-palaeodietary studies post-dating $1000 \mathrm{AD}$ in Europe ( $c f$. Barrett \& Richards 2004; Ervynck 1997; Müldner \& Richards 2005) and is a consistent pattern among ancient Christian monastic communities ( $c f$. Mays 1997; Polet \& Katzenberg 2003). 
However, it should be stressed that any isotopic signal relating to fish consumption is not necessary an indisputable link to Christian observances as regional variation and incidental exploitation of local coastal or riverine resources may also be responsible for such indications. Furthermore, the possibility that religious dietary observance by lay people took another form cannot be excluded.

\subsection{Archaeological context}

The emergence of a kingly elite and hierarchy at this time are readily visible archaeologically (Higham \& Ryan 2013). Exceptionally furnished, distinct burials appear in the funerary record, such as that at Sutton Hoo, which is an exemplar of the changing nature of social structure in Anglo-Saxon society. Hierarchy and stratification were firmly established as reflected in various grave forms. At a local level, those of apparent 'elite' status or aspiring to be, were buried under large mounds or small barrows (present at Polhill), in ship burials or at the very least were accompanied by rich goods (Lucy 2000; Welch 2011; Higham \& Ryan 2013). Although kingship appeared to be on the rise - a case perhaps of the rich getting richer - these lavish burials were not commonplace; indeed, the overall funerary patterns are characterised by fewer grave-goods than in the preceding centuries, and those which were deposited are considered 'basic' (Geake 1997; Welch 2011). The poor, it seems, were getting poorer at this time, at least on the basis of material grave wealth. The settlement record of the period appears to corroborate the burial evidence that social hierarchy was ever growing in importance (Gardiner 2011; Ulmschneider 2011).

\section{Sites}


The cemetery of Polhill dates to the late seventh and early eighth centuries, based on grave-goods typologies (Philp 1973). It is located in western Kent, near Sevenoaks, and is situated inland from the coast or nearest river (Fig. 1).

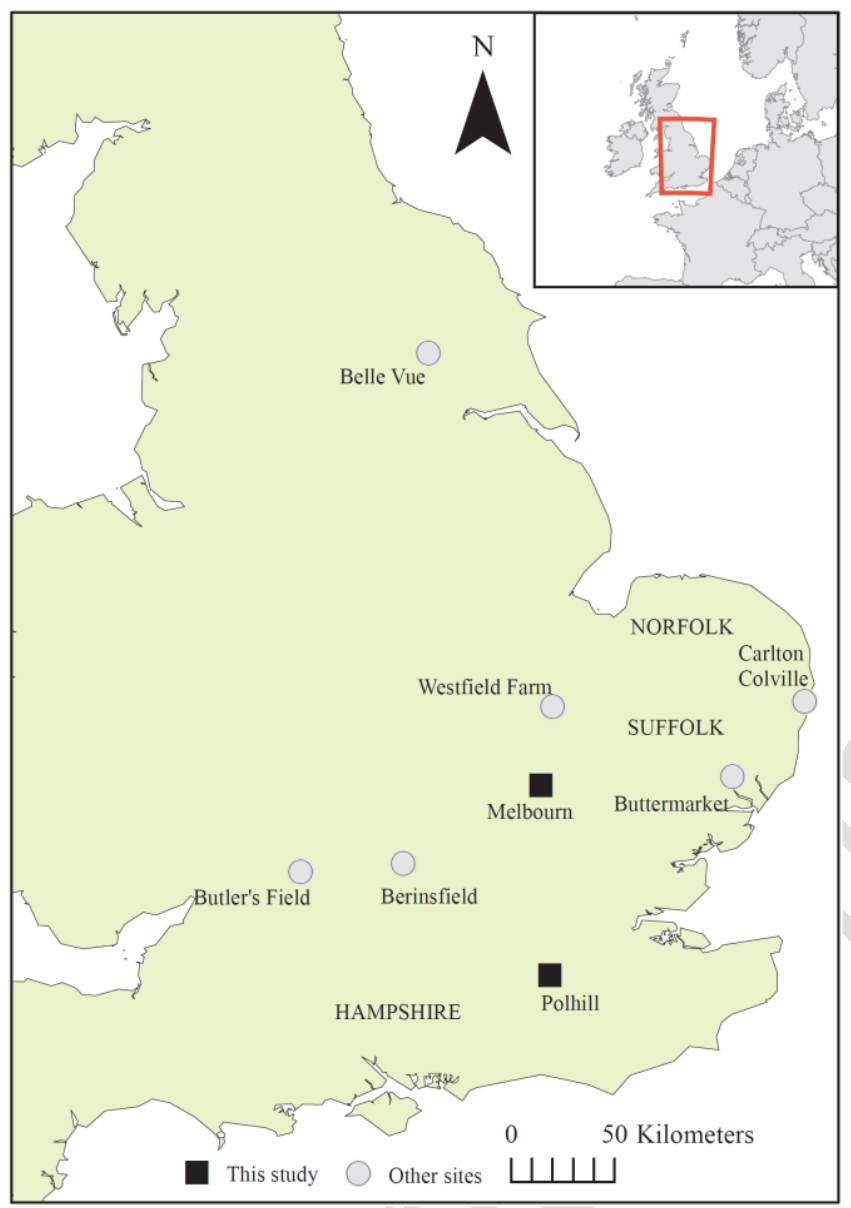

Figure 1. Map showing Polhill, Melbourn and other Anglo-Saxon cemetery locations/regions mentioned in text.

Two main areas have been excavated, producing a total of 182 burials: the west area (excavated in 1967) and the east area (excavated from 1984 to 1986), as well as some isolated finds. The Polhill individuals analysed in this study are all from the west area. Tables 1 and 2 detail information from those individuals whose age and sex could be determined (Philp 1973; Duncan et al. 2003). This part of the cemetery produced 107 inhumation graves containing over 125 skeletons (Philp 1973). Various burial forms were present, the most numerous being multiple burials, with seventeen examples identified. One contained three 
individuals, the others only two (Philp 1973). At least ten coffin burials were recorded, in addition to six barrows (with seven individuals), approximately four to five metres in diameter, which seemed to form an alignment in the north-west of the site. Grave-goods were associated with sixty-nine individuals, and at least seventeen had none. Goods included iron knives, jewellery, weaponry, dress accessories and other personal possessions (Philp 1973).

\begin{tabular}{|l|l|l|l|}
\hline & Male & Female & Indeterminate sex \\
\hline $\begin{array}{l}\text { Polhill (1967 } \\
\text { population) }\end{array}$ & 31 & 12 & 43 \\
\hline Melbourn & 23 & 17 & 9 \\
\hline
\end{tabular}

Table 1. Polhill and Melbourn sex information for adult skeletons (Philp 1973; Duncan et al. 2003).

\begin{tabular}{|l|l|l|l|}
\hline Polhill & n & Melbourn & n \\
\hline $1-6$ years & 16 & Immature & 11 \\
\hline 7-12 years & 12 & $17-25$ years & 8 \\
\hline 13-19 years & $\sim 16$ & 25-35 years & 9 \\
\hline 20-30 years & $\sim 17$ & 35-45 years & 9 \\
\hline $31-40$ years & 20 & 45+ years & 16 \\
\hline 41+ years & 19 & Indeterminate & 7 \\
\hline Other Adult & 14 & & \\
\hline Indeterminate & 11 & & \\
\hline
\end{tabular}

Table 2. Polhill and Melbourn age information, according to original age determinations (Philp 1973; Duncan et al. 2003).

The cemetery at Melbourn located south of Cambridge (Fig. 1). It was used over the sixth and seventh centuries, roughly from AD 575 to 675 (Duncan et al. 2003). Excavations in 2000, which followed earlier investigations in the area, produced sixty skeletons from fifty-five graves, alongside seven 'structures' of unknown function around which the graves seemed to gather (Wilson 1956; Duncan et al. 2003). Tables 1 and 2 quantify those individuals which could be sexed and aged. In so-called 'simple' inhumations, the skeletons displayed various body arrangements (Duncan et al. 2003). Forty graves contained grave- 
goods including dress accessories, weapons, vessels and other personal equipment (Duncan et al. 2003).

\section{Reconstructing diet using stable isotopes}

Stable isotopes of carbon and nitrogen are frequently used to investigate diet. Carbon and nitrogen isotopes ratios from bone collagen provide broad details of an individual's protein consumption (Schoeninger 1989). Broad distinctions between terrestrial and marine/freshwater resources can be made (Brown \& Brown 2011). Carbon isotope ratios distinguish between C3 (e.g. wheats, barley, etc.) and C4 (e.g. maize, millet) plants (LeeThorp 2008). The enrichment in collagen from source dietary protein is approximately 5\%o, with a further $1 \%$ enrichment in $\delta^{13} \mathrm{C}$ values with each tropic level (Malainey 2011). Nitrogen isotope ratios more clearly indicate the trophic level of an organism, with values elevated by approximately 3\%o to 5\% per trophic step in the food-chain (Lee-Thorp 2008; Schoeninger and DeNiro 1984; cf. Hull \& O'Connell 2011). This elevation in $\delta^{15} \mathrm{~N}$ would be expected with the consumption of more meat or milk products (e.g. Bocherens \& Drucker 2003; Hedges \& Reynard 2007). In the context of the current study, we therefore expect nitrogen isotope values to reflect the contribution of animal protein to diet. Both carbon and nitrogen isotopes can be used to distinguish between aquatic and terrestrial food sources (DeNiro \& Epstein 1981; Hull \& O’Connell 2011).

The relatively slow turnover rate of bone tissue ensures that dietary insights are restricted to long-term average reconstructions (Hedges et al. 2007; Lee-Thorp 2008). However, ribs for example have a faster turnover rate than long bones or teeth (Malainey 2011), thus average resolution varies according to which tissue is analysed. Bone collagen is susceptible to degradation through diagenesis (Malainey 2011), therefore it is imperative that 
the collagen quality is assessed through a series of independent checks before isotopic data can be considered reliable (section 3.2 below).

\subsection{Sample and Method}

Samples from 116 individuals were analysed: fifty-one from Melbourn and sixty-five from Polhill. The samples were prepared largely following the method outlined in Richard and Hedges (1999) but with slight modifications. One to two grams of long-bone or rib from each individual was collected. The preservation issues associated with human remains hampered the selection of a single bone element. All samples were initially cleaned by sandblasting (air-abrasion), after which $0.5 \mathrm{M}$ hydrochloric acid $(\mathrm{HCl})$ was added to each sample for demineralisation. This took two to three weeks. The samples were rinsed in distilled water and placed into $\mathrm{pH} 3.0$ water at $75^{\circ} \mathrm{C}$ for forty-eight hours for gelatinization. Following this, the gelatinized protein was filtered before being frozen and subsequently freeze-dried.

For each individual, $0.8 \pm 0.1 \mathrm{mg}$ dried collagen was analysed in triplicate, in addition to various internal and IAEA internationally referenced standards: caffeine, alanine, protein 2, nylon, and EMC. Samples were analysed in a Costech elemental analyser coupled to a Finnigan Delta V isotope-ratio mass spectrometer. The machine error was $\pm 0.1-0.2 \%$ for $\delta^{13} \mathrm{C}$ and $\delta^{15} \mathrm{~N}$. Measurements are expressed as delta $(\delta)$ values per mille $(\%): \delta^{13} \mathrm{C}$ and $\delta^{15} \mathrm{~N}$ relative to international standards VPDB (Vienna Pee Dee belemnite) and AIR (Ambiant Inhalable Reservoir), respectively (Malainey 2011). The mean and standard deviation of the triplicate $\delta^{13} \mathrm{C}, \delta^{15} \mathrm{~N}$, and $\mathrm{C}: \mathrm{N}$ ratios were calculated; errant readings from the mass spectrometer were identified as outliers and not analysed further. In these few instances, mean values were calculated based on two values. 
The sex determination for individuals remained as those originally made (Philp 1973; Duncan et al. 2003). Original age determinations were categorized into the following bands for analysis: $\mathrm{I}=$ Child, $0-14$ years $; \mathrm{I} 1=<6$ years; $\mathrm{I} 2=6-14$ years; $\mathrm{J}=14-20$ years; $\mathrm{A}=20-40$ years; $M=40-60$ years; $S=60+$ years; Ind. = Indeterminate; + (over). Wealth group determinations were made subjectively through considering the number of goods present combined with other heuristics. For example, an individual was deemed to be 'moderately wealthy' if multiple beads or a single high-quality find were present rather than the more commonplace basic iron goods. Individuals were deemed to be 'wealthy' on the basis of the quality of goods (e.g. silver or bronze), as well as number. To determine whether any differences in the isotope results between age, sex, and wealth groups were significant, statistical tests (independent t-tests and one-way ANOVA) were carried out using IBM

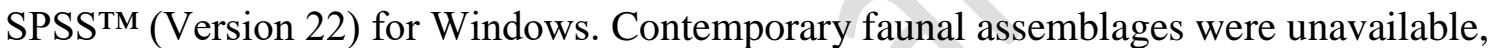
both from sites themselves and from the localities surrounding each site. Published faunal data from neighbouring regions was therefore consulted and used to interpret the results, with the proviso that isotope values are likely to vary somewhat between regions.

\subsection{Sample Preservation}

Isotopic data and associated archaeological information are summarised in Table 3 and Table 4. All samples produced an atomic $\mathrm{C}: \mathrm{N}$ ratio between 3.07-3.31, within the accepted 2.903.60 range considered to signal good collagen preservation (DeNiro 1985). Collagen yields varied although all samples produced $1.6 \%$ or more. All samples had \%C yields in excess of $13 \%$ and $\% \mathrm{~N}$ yields in excess of $4.8 \%$, indicative of well-preserved collagen (Ambrose 1990). Many were within the ranges of modern carbon (40-50\%) and modern nitrogen (1518\%) human collagen (Ambrose 1990). 


\begin{tabular}{|c|c|c|c|c|c|c|c|c|}
\hline $\begin{array}{l}\text { Sample Code (with } \\
\text { Skeleton No.) }\end{array}$ & $\operatorname{Sex}^{\mathbf{a}}$ & Age $^{b}$ & $\begin{array}{c}\text { No. } \\
\text { Grave- } \\
\text { goods }\end{array}$ & $\begin{array}{c}\text { Body } \\
\text { Arrangement }^{\mathrm{d}}\end{array}$ & $\begin{array}{c}\text { Mean } \\
{ }^{13} \mathrm{C}(\%)\end{array}$ & $\begin{array}{c}\text { Mean } \\
{ }^{15} \text { N (\%o) }\end{array}$ & $\begin{array}{c}\text { Mean } \\
\text { Atomic } \\
\text { C:N }\end{array}$ & $\begin{array}{c}\text { Collagen } \\
\text { Yield (\%) }\end{array}$ \\
\hline MEL205 & Ind. & I2 & $12 * *$ & Supine, Left & -20 & 9.6 & 3.21 & 12.1 \\
\hline MEL213 & $\mathrm{M}$ & $\mathrm{A}$ & 0 & $\begin{array}{l}\text { Supine, } \\
\text { Extended }\end{array}$ & -20.1 & 9.5 & 3.23 & 3.6 \\
\hline MEL217 & $\mathrm{M}$ & $\mathrm{A}+$ & 0 & Side, Left & -19.1 & 12 & 3.2 & 17 \\
\hline MEL305 & $\mathrm{M}$ & M+ & $3(W)$ & Supine, Crossed & -19.9 & 10.4 & 3.17 & 7.3 \\
\hline MEL312 & M & M+ & 1 & Supine, Right & -20.2 & 10.3 & 3.22 & 2.8 \\
\hline MEL316 & Ind. & $\mathrm{J}$ & 0 & Side, Left & -20.1 & 9.2 & 3.17 & 15.6 \\
\hline MEL1002 & $\mathrm{M}$ & $\mathrm{M}+$ & $7(W)$ & $\begin{array}{l}\text { Supine, } \\
\text { Extended }\end{array}$ & -20.2 & 9.4 & 3.19 & 3.2 \\
\hline MEL1008 & $\mathrm{M}$ & $\mathrm{A}$ & $3(\mathrm{~W})$ & Supine, Crossed & -20.1 & 9.7 & 3.21 & 3.8 \\
\hline MEL1012 & $M$ & $\mathrm{~A}+$ & $4(W)$ & Supine, Crossed & -20.2 & 10.1 & 3.23 & 5.8 \\
\hline MEL1015 & Ind. & I1 & 1 & $\begin{array}{l}\text { Supine, } \\
\text { Extended }\end{array}$ & -20.7 & 11.1 & 3.23 & 5.3 \\
\hline MEL1017 & $\mathrm{M}$ & $\mathrm{J}$ & 0 & $\begin{array}{c}\text { Supine, } \\
\text { Extended }\end{array}$ & -20.2 & 10.5 & 3.24 & 4.1 \\
\hline MEL1021 & $\mathrm{F}$ & A & $3 * *$ & $\begin{array}{l}\text { Supine, } \\
\text { Extended }\end{array}$ & -20.4 & 11 & 3.19 & 8.8 \\
\hline MEL1032 & $\mathrm{M}$ & $\mathrm{A}+$ & $1(\mathrm{~W})$ & $\begin{array}{l}\text { Supine, } \\
\text { Extended }\end{array}$ & -20 & 10.4 & 3.2 & 1.9 \\
\hline MEL1034 & M? & $\mathrm{A}$ & 1 & Side, Left & -20.2 & 10 & 3.17 & 11.5 \\
\hline MEL1038 & $\mathrm{F}$ & M+ & $5 * *$ & N/A & -20.1 & 9.9 & 3.17 & 7.1 \\
\hline MEL1041 & Ind. & $S$ & 0 & N/A & -20.2 & 10.3 & 3.2 & 6.5 \\
\hline MEL1045 & $\mathrm{M}$ & $\mathrm{A}$ & $5 * *$ & Supine, Left & -20.4 & 10.2 & 3.2 & 5.8 \\
\hline MEL1052 & M & A & 1 & $\begin{array}{c}\text { Supine, } \\
\text { Extended }\end{array}$ & -20.4 & 10.3 & 3.21 & 2.6 \\
\hline MEL1121 & Ind. & $\mathrm{J}+$ & 0 & N/A & -20.4 & 10.3 & 3.24 & 3.6 \\
\hline MEL1124 & M & $\mathrm{M}$ & 2 & $\begin{array}{c}\text { Supine, } \\
\text { Extended }\end{array}$ & -19.8 & 9.9 & 3.2 & 3.7 \\
\hline MEL1132 & Ind. & A & $12 * *$ & $\begin{array}{c}\text { Supine, } \\
\text { Extended }\end{array}$ & -20.3 & 9.5 & 3.21 & 3.8 \\
\hline MEL1145 & $\mathrm{M}$ & $\mathrm{A}+$ & 3 & Side, Right & -19.8 & 10.2 & 3.15 & 5.5 \\
\hline MEL1165 & $\mathrm{F}$ & $\mathrm{A}$ & $20 * *$ & $\begin{array}{l}\text { Supine, } \\
\text { Extended }\end{array}$ & -20.3 & 9.9 & 3.13 & 4.1 \\
\hline MEL1169 & F? & $\mathrm{J}+$ & 2 & $\begin{array}{l}\text { Supine, } \\
\text { Extended }\end{array}$ & -20.4 & 9.9 & 3.11 & 5.9 \\
\hline MEL1171 & $\mathrm{F}$ ? & Ind. & 0 & N/A & -20.1 & 10.1 & 3.13 & 5.6 \\
\hline MEL1174 & Ind. & Ind. & 0 & N/A & -20.4 & 9.8 & 3.12 & 3.3 \\
\hline MEL1176 & $\mathrm{F}$ & M+ & $1 * *$ & $\begin{array}{l}\text { Supine, } \\
\text { Extended }\end{array}$ & -20.1 & 9.4 & 3.18 & 5.4 \\
\hline MEL1184 & $\mathrm{M}$ & $\mathrm{M}$ & 2 & $\begin{array}{l}\text { Supine, } \\
\text { Extended }\end{array}$ & -20.3 & 9.8 & 3.16 & 4.7 \\
\hline MEL1187 & $\mathrm{M}$ & $S$ & 5 & $\begin{array}{c}\text { Supine, } \\
\text { Extended }\end{array}$ & -20 & 9.7 & 3.14 & 13.1 \\
\hline MEL1188 & $\mathrm{M}$ & $S$ & 2 & Supine, Crossed & -20 & 9.8 & 3.11 & 6.3 \\
\hline MEL1189 & $\mathrm{F}$ & $\mathrm{A}+$ & $2 * *$ & Side, Left & -20.2 & 9.4 & 3.13 & 3.2 \\
\hline MEL1199 & $\mathrm{F}$ & Ind. & 1 & N/A & -20.3 & 11.4 & 3.11 & 7.8 \\
\hline MEL1204 & $\mathrm{M}$ & $\mathrm{J}$ & $3(W)$ & $\begin{array}{c}\text { Supine, } \\
\text { Extended }\end{array}$ & -20.2 & 9.1 & 3.12 & 6.1 \\
\hline MEL1224 & Ind. & I2 & 2 & Side, Left & -20.1 & 8.8 & 3.18 & 3.3 \\
\hline MEL1229 & $\mathrm{F}$ & $\mathrm{J}+$ & $15 * *$ & $\begin{array}{l}\text { Supine, } \\
\text { Extended }\end{array}$ & -20.2 & 10.3 & 3.12 & 5.4 \\
\hline MEL1259 & $\mathrm{F}$ & $\mathrm{A}$ & 0 & N/A & -20.3 & 9.4 & 3.1 & 6.3 \\
\hline MEL1263 & F? & $\mathrm{J}+$ & 2 & Supine, Left & -20.2 & 10.3 & 3.12 & 10 \\
\hline
\end{tabular}




\begin{tabular}{|c|c|c|c|c|c|c|c|c|}
\hline $\begin{array}{c}\text { Sample Code (with } \\
\text { Skeleton No.) }\end{array}$ & Sex $^{\mathbf{a}}$ & Age $^{\mathbf{b}}$ & $\begin{array}{c}\text { No. } \\
\mathbf{G r a v e -} \\
\mathbf{g o o d s}^{\mathbf{c}}\end{array}$ & $\begin{array}{c}\text { Body } \\
\text { Arrangement }\end{array}$ & $\begin{array}{c}\text { Mean } \\
\mathbf{1 3} \mathbf{C} \text { (\%) }\end{array}$ & $\begin{array}{c}\text { Mean } \\
\mathbf{1 5} \mathbf{N} \text { (\%) }\end{array}$ & $\begin{array}{c}\text { Mean } \\
\text { Atomic } \\
\text { C:N }\end{array}$ & $\begin{array}{c}\text { Collagen } \\
\text { Yield (\%) }\end{array}$ \\
\hline MEL1267 & F & J & $4^{*}$ & $\begin{array}{c}\text { Supine, } \\
\text { Extended }\end{array}$ & -20.4 & 9.3 & 3.13 & 5.1 \\
\hline MEL1293 & Ind. & A & $14^{* *}$ & Supine, Left & -20.3 & 9.4 & 3.15 & 3.9 \\
\hline MEL1305 & F & S & 0 & Supine, Crossed & -19.9 & 9.9 & 3.13 & 5 \\
\hline MEL1307 & F? & M+ & $76^{* *}$ & Supine, Crossed & -20.6 & 9.6 & 3.19 & 2.4 \\
\hline MEL1312 & M & A+ & 0 & $\begin{array}{c}\text { Supine, } \\
\text { Extended }\end{array}$ & -19.8 & 10 & 3.13 & 4 \\
\hline MEL1321 & Ind. & J+ & 2 & Side, Left & -20.2 & 9.4 & 3.19 & 2.3 \\
\hline MEL1322 & M? & Ind. & 3 & N/A & -20.1 & 9.8 & 3.13 & 3.4 \\
\hline MEL1325 & Ind. & J+ & $6^{*}$ & N/A & -20.2 & 9.7 & 3.19 & 3 \\
\hline MEL1348 & M & A+ & 0 & $\begin{array}{c}\text { Supine, } \\
\text { Extended }\end{array}$ & -19.6 & 9.6 & 3.16 & 5.4 \\
\hline MEL1370 & F? & A & $14^{* *}$ & $\begin{array}{c}\text { Supine, } \\
\text { Extended }\end{array}$ & -20.3 & 10 & 3.21 & 3.2 \\
\hline MEL1386 & M & A+ & 2 & $\begin{array}{c}\text { Supine, } \\
\text { Extended }\end{array}$ & -20.1 & 9 & 3.15 & 7.2 \\
\hline MEL1388 & M & A & 0 & N/A & -20.3 & 10.3 & 3.14 & 2.6 \\
\hline MEL1398 & F & M+ & $4^{*}$ & Side, Left & -20.1 & 9.7 & 3.13 & 12.4 \\
\hline MEL1400 & Ind. & I1 & 1 & N/A & -20.6 & 10.8 & 3.25 & 2.5 \\
\hline
\end{tabular}

Table 3. Melbourn human archaeological information and isotopic data. Key: $\mathbf{A}=$ Sex:

$\mathrm{M}=$ male; $\mathrm{F}=$ female; $\mathrm{F}$ ?=possible female; $\mathrm{M}$ ?=possible male; Ind.=indeterminate. $\mathbf{B}=$ Age: $\mathrm{I}=$ Child, 0-14years; $\mathrm{I} 1=<6$ years; $\mathrm{I} 2=6-14$ years; $\mathrm{J}=14-20$ years; $\mathrm{A}=20-40$ years; $\mathrm{M}=40$ 60 years; $S=60+$ years; Ind. = Indeterminate; + (over). $C=$ Number of grave-goods: Classified by number present and subjective decision-making. 'Moderately wealthy' deemed on, e.g. multiple beads present or a single high-quality find present; not simply iron goods present. 'Wealthy' deemed mainly on quality, as well as number, e.g. silver or numerous beads, bronze present. $*=$ moderately wealthy goods; $* *=$ wealthy goods; $\mathrm{W}=$ weapon(s) present. D=Body Arrangement: Supine or Side; with legs: extended, crossed at ankles, bent left, or bent right (following Duncan et al. 2003). 


\begin{tabular}{|c|c|c|c|c|c|c|c|c|}
\hline $\begin{array}{c}\text { Sample Code } \\
\text { (with Box/Grave } \\
\text { No.) }\end{array}$ & Sex $^{a}$ & Age $^{b}$ & $\begin{array}{c}\text { No. } \\
\text { Grave- } \\
\text { goods }\end{array}$ & $\begin{array}{l}\text { Grave } \\
\text { Type }^{\text {e }}\end{array}$ & $\begin{array}{c}\text { Mean } \\
\delta^{13} \mathrm{C}(\%)\end{array}$ & $\begin{array}{c}\text { Mean } \\
\delta^{15} \mathbf{N}(\%)\end{array}$ & $\begin{array}{c}\text { Mean } \\
\text { Atomic } \\
\text { C:N }\end{array}$ & $\begin{array}{l}\text { Collagen } \\
\text { Yield }(\%)\end{array}$ \\
\hline POL2 & Ind. & I & 0 & Cut & -20.0 & 9.4 & 3.20 & 5.1 \\
\hline POL4 & $\mathrm{M}$ & $\mathrm{A}+$ & 0 & Cut & -20.2 & 8.1 & 3.31 & 3.2 \\
\hline POL5 & $\mathrm{M}$ & A & 0 & Cut & -20.3 & 11.0 & 3.26 & 9.1 \\
\hline POL10 & Ind. & $\mathrm{A}+$ & 0 & Cut & -20.8 & 10.3 & 3.31 & 1.8 \\
\hline POL36 & Ind. & $\mathrm{A}$ & 0 & Cut & -20.4 & 9.7 & 3.18 & 3.0 \\
\hline POL38 & F? & A & $6 * *$ & Cut & -20.2 & 8.7 & 3.13 & 14.3 \\
\hline POL39 & $\mathrm{F}$ & $\mathrm{A}$ & 2 & Cut & -20.4 & 10.9 & 3.18 & 6.0 \\
\hline POL40 & $\mathrm{M}$ & $\mathrm{J}$ & $3(W)$ & Cut & -20.1 & 10.1 & 3.17 & 18.5 \\
\hline POL41 & M? & Ind. & $14 * *$ & Cut & -20.4 & 8.9 & 3.18 & 20.0 \\
\hline POL42N & M? & $\mathrm{A}$ & 1 & Multiple & -20.6 & 9.3 & 3.24 & 2.7 \\
\hline POL42S & M? & $\mathrm{J}$ & 1 & Multiple & -20.7 & 9.4 & 3.20 & 4.7 \\
\hline POL43N & F? & $\mathrm{A}+$ & $6 * *$ & Multiple & -21.0 & 9.2 & 3.21 & 4.8 \\
\hline POL43S & $\mathrm{F}$ & $\mathrm{J}+$ & 0 & Multiple & -20.7 & 9.4 & 3.19 & 4.3 \\
\hline POL44 & F? & A & 1 & Coffin & -20.2 & 8.7 & 3.11 & 6.8 \\
\hline POL45 & $\mathrm{M}$ & $\mathrm{M}$ & $4(W)$ & Cut & -20.2 & 10.5 & 3.17 & 19.8 \\
\hline POL46 & Ind. & $\mathrm{A}+$ & 0 & Cut & -20.6 & 9.6 & 3.17 & 7.6 \\
\hline POL47 & $\mathrm{F}$ & $\mathrm{A}$ & 0 & Coffin & -20.3 & 9.5 & 3.23 & 10.6 \\
\hline POL52N & F? & $\mathrm{A}$ & 1 & Multiple & -20.2 & 10.4 & 3.27 & 3.6 \\
\hline POL52S & Ind. & $\mathrm{A}$ & 1 & Multiple & -20.4 & 9.5 & 3.13 & 4.6 \\
\hline POL53 & F? & $\mathrm{A}+$ & $5^{*}$ & Cut & -20.3 & 10.6 & 3.09 & 3.0 \\
\hline POL56 & Ind. & $\mathrm{J}$ & 1 & Cut & -20.5 & 9.2 & 3.23 & 3.5 \\
\hline POL57 & Ind. & I2 & 1 & Cut & -20.7 & 9.0 & 3.21 & 7.4 \\
\hline POL60L & Ind. & $\mathbf{J}$ & 1 & Multiple & -20.4 & 9.3 & 3.21 & 1.7 \\
\hline POL60U & $\mathrm{F}$ & $\mathrm{A}+$ & 0 & Multiple & -20.1 & 9.3 & 3.25 & 3.9 \\
\hline POL61 & $\mathrm{M}$ & $\mathrm{A}$ & 1 & Cut & -20.5 & 9.9 & 3.23 & 2.5 \\
\hline POL62 & Ind. & Ind. & 2 & Cut & -20.3 & 9.9 & 3.17 & 16.0 \\
\hline POL64N & Ind. & Ind. & 2 & Multiple & -20.6 & 10.2 & 3.24 & 5.1 \\
\hline POL64S & M? & $\mathrm{A}+$ & $1 * *$ & Multiple & -20.4 & 9.8 & 3.22 & 10.7 \\
\hline POL65 & $\mathrm{M}$ & $\mathrm{A}$ & $6^{* *}(\mathrm{~W})$ & Cut & -20.2 & 9.1 & 3.17 & 9.7 \\
\hline POL67 & Ind. & $\mathrm{A}+$ & 0 & Cut & -20.3 & 10.6 & 3.15 & 18.5 \\
\hline POL68(N) & M? & $\mathrm{A}$ & $4(W)$ & Multiple & -20.3 & 9.6 & 3.15 & 5.8 \\
\hline POL68S & Ind. & $\mathrm{J}$ & 2 & Multiple & -20.7 & 9.9 & 3.21 & 5.8 \\
\hline POL69N & Ind. & I & 1 & Multiple & -20.4 & 9.4 & 3.12 & 2.8 \\
\hline POL69S & M? & $\mathrm{J}$ & $2(W)$ & Multiple & -20.2 & 9.7 & 3.20 & 5.9 \\
\hline POL71 & F? & $\mathrm{J}$ & $8 *$ & Cut & -20.5 & 9.7 & 3.14 & 19.2 \\
\hline POL72 & Ind. & $\mathrm{I} 2$ & 1 & Cut & -20.6 & 11.0 & 3.09 & 10.5 \\
\hline POL73 & $\mathrm{M}$ & $\mathrm{A}$ & $2(W)$ & Cut & -20.9 & 9.0 & 3.30 & 8.2 \\
\hline POL75N & Ind. & $\mathrm{I} 2$ & 1 & Multiple & -20.3 & 8.8 & 3.17 & 8.7 \\
\hline POL75S & F? & $\mathrm{A}$ & 3 & Multiple & -20.3 & 9.0 & 3.17 & 3.6 \\
\hline POL76 & $\mathrm{F}$ & $\mathrm{A}$ & 0 & Coffin & -20.3 & 9.1 & 3.15 & 2.04 \\
\hline
\end{tabular}




\begin{tabular}{|c|c|c|c|c|c|c|c|c|}
\hline $\begin{array}{l}\text { Sample Code } \\
\text { (with Box/Grave } \\
\text { No.) }\end{array}$ & $\operatorname{Sex}^{\mathbf{a}}$ & Age $^{b}$ & $\begin{array}{c}\text { No. } \\
\text { Grave- } \\
\text { goods }\end{array}$ & $\begin{array}{l}\text { Grave } \\
\text { Type }^{\mathrm{e}}\end{array}$ & $\begin{array}{c}\text { Mean } \\
\delta^{13} \mathrm{C}(\%)\end{array}$ & $\begin{array}{c}\text { Mean } \\
\delta^{15} \mathbf{N}(\%)\end{array}$ & $\begin{array}{c}\text { Mean } \\
\text { Atomic } \\
\mathrm{C}: \mathbf{N}\end{array}$ & $\begin{array}{c}\text { Collagen } \\
\text { Yield }(\%)\end{array}$ \\
\hline POL77 & M & Ind. & 2 & Coffin & -19.9 & 11.1 & 3.18 & 1.6 \\
\hline POL81N & Ind. & Ind. & 0 & Multiple & -20.1 & 9.0 & 3.18 & 15.6 \\
\hline POL82N & Ind. & $\mathrm{I}$ & 1 & Multiple & -20.6 & 9.6 & 3.19 & 3.3 \\
\hline POL82S & $\mathrm{F}$ & $\mathrm{J}+$ & 0 & Multiple & -20.4 & 9.4 & 3.16 & 6.7 \\
\hline POL83 & Ind. & $\mathrm{A}+$ & $2 *$ & Cut & -20.3 & 13.2 & 3.11 & 7.8 \\
\hline POL84 & M? & Ind. & $5(W)$ & Cut & -20.4 & 8.9 & 3.15 & 9.5 \\
\hline POL85 & M? & $\mathrm{J}+$ & $8^{*}(\mathrm{~W})$ & Cut & -20.0 & 10.9 & 3.21 & 1.8 \\
\hline POL86N & Ind. & Ind. & 0 & Multiple & -20.4 & 9.7 & 3.14 & 4.3 \\
\hline POL86S & M? & $\mathrm{A}+$ & 3 & Multiple & -20.2 & 9.8 & 3.15 & 7.5 \\
\hline POL87 & Ind. & Ind. & 0 & Cut & -20.2 & 8.8 & 3.19 & 1.9 \\
\hline POL88 & Ind. & $\mathrm{I} 2$ & 1 & $\begin{array}{c}\text { Coffin } \\
\text { (possible) }\end{array}$ & -20.5 & 9.4 & 3.18 & 6.4 \\
\hline POL89 & $\mathrm{M}$ & Ind. & 2 & Cut & -20.2 & 9.4 & 3.11 & 6.1 \\
\hline POL90 & M? & Ind. & 2 & Cut & -20.5 & 9.4 & 3.21 & 8.2 \\
\hline POL91 & $\mathrm{M}$ & $\mathrm{A}$ & 1 & $\begin{array}{c}\text { Barrow, } \\
\text { Coffin }\end{array}$ & -20.0 & 10.5 & 3.17 & 20.6 \\
\hline POL92 & $\mathrm{F}$ & A & 0 & Coffin & -20.2 & 10.6 & 3.23 & 1.6 \\
\hline POL93 & $\mathrm{F}$ & $\mathrm{J}+$ & 1 & Barrow & -20.4 & 10.2 & 3.09 & 4.2 \\
\hline POL95 & Ind. & $\mathrm{I} 2$ & $7 *$ & Barrow & -20.7 & 9.7 & 3.09 & 16.8 \\
\hline POL97 & Ind. & $\mathrm{J}$ & $4(W)$ & Cut & -20.6 & 9.8 & 3.12 & 3.5 \\
\hline POL98 & M? & $\mathrm{M}$ & 1 & $\begin{array}{l}\text { Barrow, } \\
\text { Coffin }\end{array}$ & -20.1 & 10.2 & 3.07 & 16.4 \\
\hline POL99N & $\mathrm{F}$ & A & 0 & Multiple & -20.5 & 9.3 & 3.21 & 6.5 \\
\hline POL99C & $\mathrm{M}$ & $\mathrm{A}$ & 2 & Multiple & -20.3 & 9.1 & 3.11 & 8.0 \\
\hline POL99S & Ind. & $\mathrm{A}+$ & 0 & Multiple & -20.8 & 9.4 & 3.21 & 4.8 \\
\hline POL100 & Ind. & $\mathrm{I} 2$ & 0 & Cut & -20.6 & 11.0 & 3.19 & 4.2 \\
\hline POL102N & Ind. & Ind. & $6^{*}(\mathrm{~W})$ & Multiple & -20.4 & 9.6 & 3.16 & 4.0 \\
\hline POL102S & F & Ind. & $17 * *$ & Multiple & -20.5 & 9.3 & 3.14 & 2.5 \\
\hline
\end{tabular}

Table 4. Polhill human archaeological information and isotopic data. Key: $\mathbf{A}, \mathbf{B}, \mathbf{C}$ (see Table 1 key). $\mathbf{E}$ - Grave type: Cut = simple, rectangular, cut grave; Multiple = two or more skeletons present in single grave; Barrow $=$ ditched barrow surrounding grave Coffin $=$ coffin/coffin traces present.

\section{Results}

\subsection{Overall diet}

Biplots of human isotopic data for Melbourn and Polhill appear in Figures 2 and 3 respectively. On average, the individuals from Melbourn had slightly more enriched $\delta^{13} \mathrm{C}$ 
values then those from Polhill, which was statistically significant ( $\mathrm{t}$-test, $t=5.063, \mathrm{df}=114, \mathrm{p}$

$\left.\sim 10^{-6}\right)$.

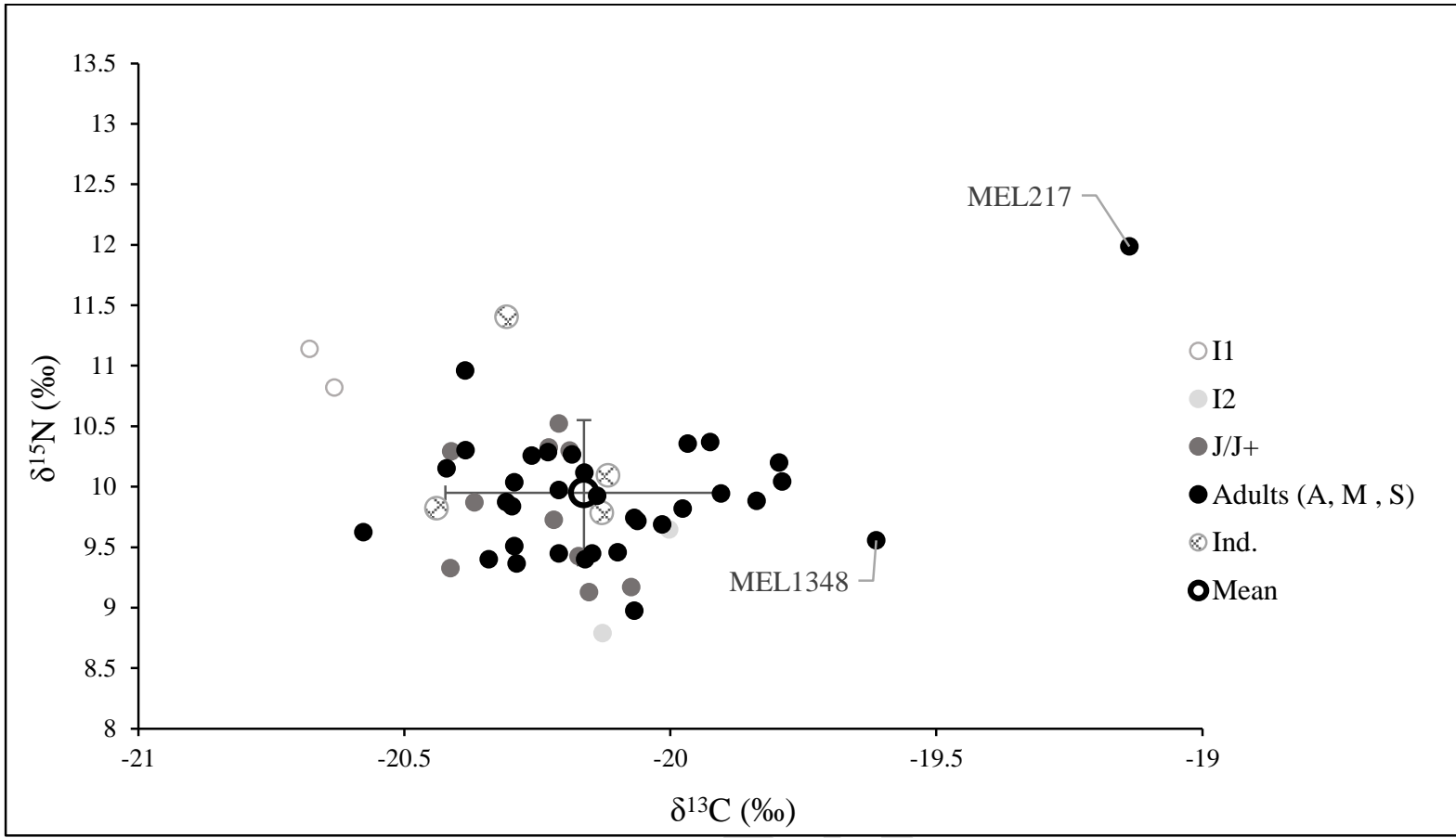

Figure 2. Melbourn human isotopic values, with mean and standard deviations. Data also differentiated according to age.

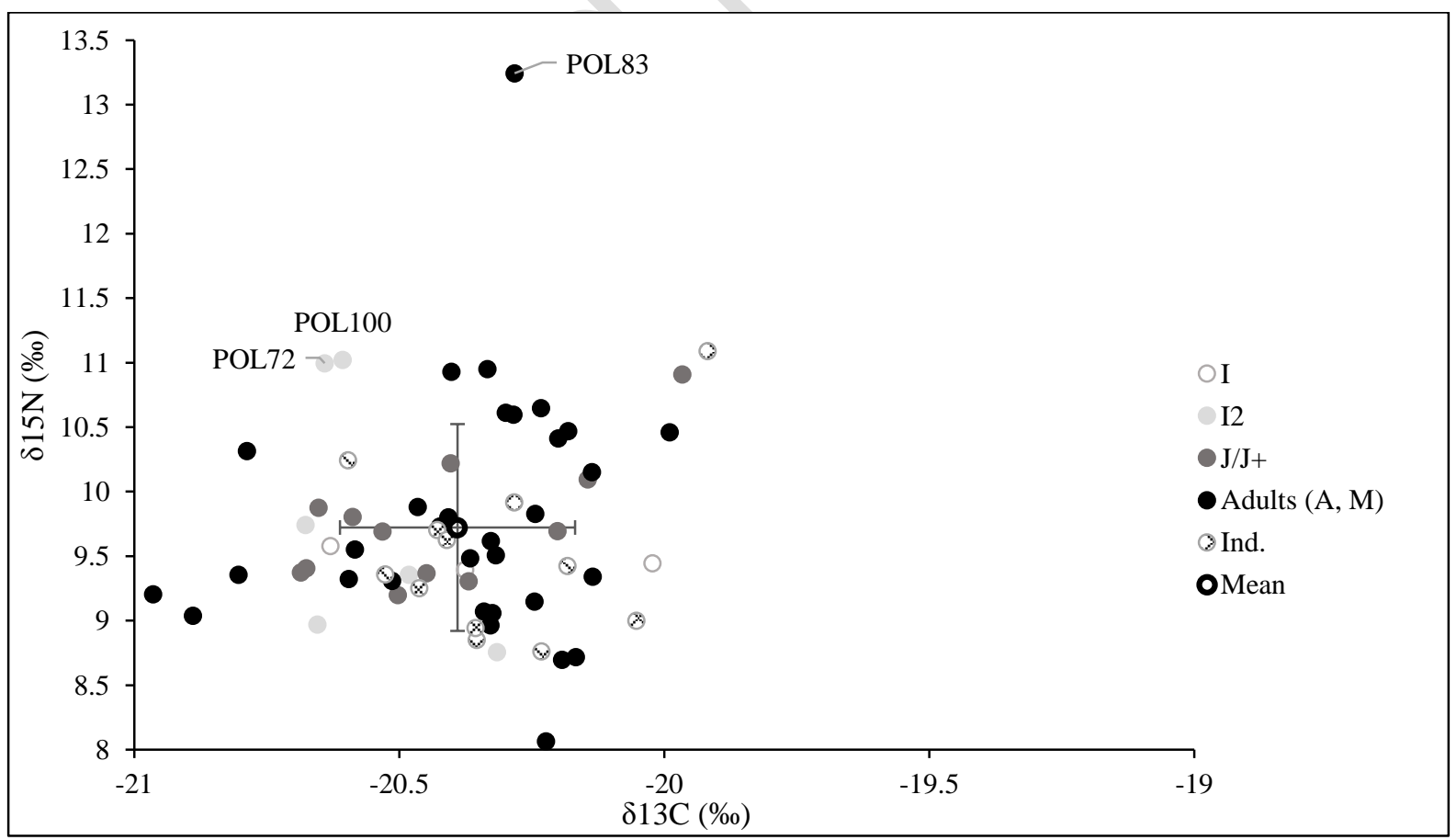

Figure 3. Polhill human isotopic values, with mean and standard deviations. Data also differentiated according to age. 
Generally, the Melbourn diet appears to have been largely uniform within the population sample, with only one clear outlier (MEL217). The sample had limited $\delta^{13} \mathrm{C}$ variability (Table 5), with most individuals tightly clustered between $-20.4 \%$ and $-19.8 \%$. The overall picture suggests a diet based mainly on $\mathrm{C}_{3}$ terrestrial plants with regular consumption of animal protein by all individuals, and no marine intake (Chisholm et al. 1982; Schoeninger \& DeNiro 1984). The possibility that freshwater fish were consumed cannot be excluded, particularly for those individuals with $\delta^{15} \mathrm{~N}$ values greater than $10.5 \%$. The source values of such fish range from 6.6 to $9.5 \%$ for $\delta^{15} \mathrm{~N}$ and -23.7 to -12.7 for $\delta^{13} \mathrm{C}$ (Schoeninger \& DeNiro 1984), although these values can vary considerably according to location (Dufour et al. 1999) and species (Keaveney et al. 2015). Alternatively, and more likely given to Melbourn's location inland, individuals with $\delta^{15} \mathrm{~N}$ above $10.5 \%$ may have consumed more terrestrial animal protein (i.e. meat or dairy) than others in the group. Whether this is due to differences in social rank is discussed further below.

\begin{tabular}{|l|l|l|l|l|l|l|l|}
\hline Site & $\begin{array}{l}\text { Mean } \\
\text { (\%o) }\end{array}$ & $\begin{array}{l}\boldsymbol{\delta}^{\mathbf{1 3}} \mathbf{C} \\
\mathbf{( \% o} \\
(\mathbf{m i n} \\
\mathbf{m a x})\end{array}$ & Range & $\begin{array}{l}\text { Mean } \\
(\mathbf{\% o})\end{array}$ & $\begin{array}{l}\boldsymbol{\delta}^{\mathbf{1 5}} \mathbf{N} \\
\mathbf{( \% o} \\
(\mathbf{m i n} ; \\
\mathbf{m a x} .)\end{array}$ & Range & $n$ \\
\hline Melbourn & -20.2 & $\begin{array}{l}(-20.7 ;- \\
19.1)\end{array}$ & 1.6 & 10.0 & $\begin{array}{l}(8.8 ; \\
12.0\end{array}$ & 3.2 & 51 \\
\hline Polhill & -20.4 & $\begin{array}{l}(-21.0 ;- \\
19.9)\end{array}$ & 1.1 & 9.7 & $\begin{array}{l}(8.1 ; \\
13.2)\end{array}$ & 5.1 & 65 \\
\hline
\end{tabular}

Table 5. Mean and range data for $\delta^{13} \mathrm{C}$ and $\delta^{15} \mathrm{~N}$ values from Melbourn and Polhill individuals.

At Polhill, individuals exhibited a narrower $\delta^{13} \mathrm{C}$ range than Melbourn, but the $\delta^{15} \mathrm{~N}$ range is somewhat broader (Table 5). The individual POL83 has a particularly enriched $\delta^{15} \mathrm{~N}$ value compared to the site average, differing by approximately $2.1 \%$, suggesting that this individual consumed much more animal protein. A small number of individuals with relatively depleted $\delta^{13} \mathrm{C}$ and $\delta^{15} \mathrm{~N}$ values, could indicate a diet based mostly on plant-based foods, with little animal protein input. However, the overall picture is one of homogeneous, temperate, terrestrial-based mixed diet of $\mathrm{C} 3$ plants with meat and/or diary featuring in the 
diet, as well as possible freshwater sources. Generally, the diet at Polhill was quite uniform but the broader $\delta^{15} \mathrm{~N}$ range implies that some individuals consumed more animal protein than others, as would be expected in a society where diet was linked in some way to social rank.

\section{Diet and Social Structure}

\begin{tabular}{|c|c|c|c|c|c|c|c|}
\hline & \multicolumn{3}{|c|}{ Melbourn } & & & \multicolumn{2}{|c|}{ Polhill } \\
\hline & $\mathbf{n}$ & $\begin{array}{c}\text { Mean } \delta^{13} C \\
\pm \text { sd (\%o) }\end{array}$ & $\begin{array}{c}\text { Mean } \delta^{15} \mathbf{N} \\
\pm \operatorname{sd}(\% o)\end{array}$ & & $\mathbf{n}$ & $\begin{array}{c}\text { Mean } \delta^{13} C \\
\pm \text { sd (\%o) }\end{array}$ & $\begin{array}{c}\text { Mean } \delta^{15} N \\
\pm \text { sd (\%o) }\end{array}$ \\
\hline \multicolumn{8}{|c|}{ Sex } \\
\hline Male & 23 & $\begin{array}{l}-20.0 \\
\pm 0.28\end{array}$ & $10.0 \pm 0.59$ & Male & 22 & $-20.3 \pm 0.23$ & $9.7 \pm 0.76$ \\
\hline Female & 16 & $\begin{array}{l}-20.2 \\
\pm 0.16 \\
\end{array}$ & $10.0 \pm 0.56$ & Female & 17 & $-20.4 \pm 0.21$ & $9.6 \pm 0.70$ \\
\hline Ind. & 12 & $\begin{array}{l}-20.3 \\
\pm 0.21\end{array}$ & $9.8 \pm 0.69$ & Ind. & 26 & $-20.5 \pm 0.20$ & $9.8 \pm 0.92$ \\
\hline \multicolumn{8}{|c|}{ Age } \\
\hline I1 & 2 & $\begin{array}{l}-20.7 \\
\pm 0.03\end{array}$ & $11.0 \pm 0.22$ & $\mathrm{I} / \mathrm{I} 2$ & 9 & $-20.5 \pm 0.22$ & $9.7 \pm 0.80$ \\
\hline $\mathrm{I} 2$ & 2 & $\begin{array}{l}-20.1 \\
\pm 0.08\end{array}$ & $9.2 \pm 0.61$ & r & & & \\
\hline $\mathrm{J} / \mathrm{J}+$ & 10 & $\begin{array}{l}-20.2 \\
\pm 0.12\end{array}$ & $9.8 \pm 0.53$ & $\mathrm{~J} / \mathrm{J}+$ & 12 & $-20.4 \pm 0.23$ & $9.7 \pm 0.49$ \\
\hline $\begin{array}{l}\text { Adults (A, } \\
M, S)\end{array}$ & 33 & $\begin{array}{l}-20.1 \\
\pm 0.26\end{array}$ & $9.9 \pm 0.60$ & $\begin{array}{l}\text { Adults (A, } \\
\text { M) }\end{array}$ & 32 & $-20.4 \pm 0.22$ & $9.8 \pm 0.80$ \\
\hline Ind. & 4 & $\begin{array}{l}-20.2 \\
\pm 0.15\end{array}$ & $10.3 \pm 0.77$ & Ind. & 12 & $-20.3 \pm 0.20$ & $9.5 \pm 0.67$ \\
\hline \multicolumn{8}{|c|}{ Wealth } \\
\hline None & 13 & $\begin{array}{l}-20.0 \\
\pm 0.36\end{array}$ & $10.1 \pm 0.71$ & None & 19 & $-20.4 \pm 0.23$ & $9.6 \pm 0.76$ \\
\hline 5 or less & 22 & $\begin{array}{l}-20.2 \\
\pm 0.22\end{array}$ & $10.0 \pm 0.63$ & 5 or less & 34 & $-20.4 \pm 0.21$ & $9.7 \pm 0.64$ \\
\hline 6 to 10 & 1 & -20.2 & 9.4 & & & & \\
\hline $\begin{array}{l}\text { Moderately } \\
\text { wealthy }\end{array}$ & 3 & $\begin{array}{l}-20.2 \\
\pm 0.17 \\
\end{array}$ & $9.6 \pm 0.24$ & $\begin{array}{l}\text { Moderately } \\
\text { wealthy }\end{array}$ & 6 & $-20.4 \pm 0.24$ & $10.6 \pm 1.38$ \\
\hline Wealthy & 12 & $\begin{array}{l}-20.3 \\
\pm 0.15\end{array}$ & $9.9 \pm 0.46$ & Wealthy & 6 & $-20.4 \pm 0.28$ & $9.2 \pm 0.38$ \\
\hline Weapons & 6 & $\begin{array}{l}-20.1 \\
\pm 0.10\end{array}$ & $9.8 \pm 0.52$ & Weapons & 10 & $-20.3 \pm 0.26$ & $9.7 \pm 0.63$ \\
\hline \multicolumn{8}{|c|}{ Burial Feature } \\
\hline None & 11 & $\begin{array}{l}-20.3 \\
\pm 0.16\end{array}$ & $10.2 \pm 0.56$ & Multiple & 26 & $-20.4 \pm 0.21$ & $9.5 \pm 0.37$ \\
\hline
\end{tabular}




\begin{tabular}{|l|l|l|l|l|l|l|l|}
\hline Side, Left & 7 & $\begin{array}{l}-20.0 \\
\pm 0.38\end{array}$ & $9.8 \pm 1.04$ & & & & \\
\hline Side, Right & 1 & -19.8 & 10.2 & Cut & 29 & $-20.4 \pm 0.22$ & $9.9 \pm 1.03$ \\
\hline $\begin{array}{l}\text { Supine, } \\
\text { Crossed }\end{array}$ & 6 & $\begin{array}{l}-20.1 \\
\pm 0.25\end{array}$ & $9.9 \pm 0.28$ & & & & \\
\hline $\begin{array}{l}\text { Supine, } \\
\text { Extended }\end{array}$ & 21 & $\begin{array}{l}-20.2 \\
\pm 0.24\end{array}$ & $9.9 \pm 0.56$ & Coffin & 6 & $-20.2 \pm 0.19$ & $9.7 \pm 0.94$ \\
\cline { 1 - 4 } & 4 & $\begin{array}{l}-20.2 \\
\pm 0.17\end{array}$ & $9.9 \pm 0.44$ & & & & \\
\cline { 1 - 4 } $\begin{array}{l}\text { Supine, } \\
\text { Right }\end{array}$ & 1 & -20.2 & 10.3 & $\begin{array}{l}\text { Barrow, } \\
\text { Coffin }\end{array}$ & 2 & $-20.1 \pm 0.10$ & $10.3 \pm 0.22$ \\
\cline { 3 - 6 } & & Barrow & 2 & $-20.5 \pm 0.19$ & $10.0 \pm 0.34$ \\
\hline
\end{tabular}

Table 6. Mean $\delta^{13} \mathrm{C}$ and $\delta^{15} \mathrm{~N}$ values (with standard deviations) for Melbourn and Polhill samples according to sex, age, grave-good wealth, and burial feature.

\subsection{Sex}

The Melbourn data display similar $\delta^{13} \mathrm{C}$ and $\delta^{15} \mathrm{~N}$ ranges when grouped by sex, indicating little sex-based dietary division (see Table 6). The mean $\delta^{15} \mathrm{~N}$ results for males $(n=23)$ and females $(n=16)$ are identical $(10.0 \%)$, with that of indeterminate sex being closely similar (9.8\%o). The $\delta^{13} \mathrm{C}$ values show a slight (by .2-.3\%o) difference between males and females which was statistically significant ( $\mathrm{t}$-test, $t=2.20, \mathrm{df}=30, p=.036$ ). There are two male individuals whose values deviate significantly from the rest: MEL217 (in $\delta^{13} \mathrm{C}$ and $\delta^{15} \mathrm{~N}$ ) and MEL1348 (in $\delta^{13} \mathrm{C}$ ). MEL217 probably consumed more animal protein and both displayed less negative $\delta^{13} \mathrm{C}$ values than the main group. Both are adults (20 to $40+$ years) with no grave-goods. The values at Polhill (Table 6) showed no significant sex-dependent dietary differences. This reflects the results of previous work at other sites, such as Berinsfield, Oxfordshire, and Carlton Colville, Suffolk (Privat et al. 2002; O'Connell \& Lawler 2009). The most notable outlier, POL83, which had particularly enriched ${ }^{15} \mathrm{~N}$, was an adult of indeterminate sex buried with only moderately wealthy grave-goods. Like the individuals from Melbourn, there was no outstanding archaeological feature that could have predicted this individual's unusual diet. It is possible, but not otherwise demonstrable, that 
POL83 as well as the two Melbourn outliers (MEL217 and MEL 1348) were non-locals within both cemetery populations.

\subsection{Age}

Age-dependent differences between children and adults were evident at Melbourn, (Fig. 2). Table 6 includes the mean values for $\delta^{13} \mathrm{C}$ and $\delta^{15} \mathrm{~N}$ for both sites according to age. The $\mathrm{A} / \mathrm{A}+, \mathrm{M} / \mathrm{M}+$ and $\mathrm{S}$ age categories were considered as one adult group $(\mathrm{n}=33)$. An ANOVA test showed statistically significant differences in $\delta^{13} \mathrm{C}(\mathrm{F}(3,43)=3.847, \mathrm{p} .016)$ and in $\delta^{15} \mathrm{~N}(\mathrm{~F}(3,43)=3.918, \mathrm{p}=.015)$ between the adult, juvenile $(\mathrm{n}=10)$ and child $(\mathrm{n}=4)$ groups. The relatively small number of children in the study restricts the feasibility taking this analysis further. Post-hoc Bonferroni tests illustrate that the I1 and Adults group $\delta^{13} \mathrm{C}$ differed $(p=0.019)$, as did the I1 and $\mathrm{I} 2$ and $\mathrm{I} 1$ and $\mathrm{J} / \mathrm{J}+\delta^{15} \mathrm{~N}$ values $(\mathrm{p}=.0 .12$ and $\mathrm{p}=.043$ respectively), although as the I1 and $\mathrm{I} 2$ groups have only two individuals, these tests could be misleading. Nevertheless, these results conform to the typical patterns expected due to biological differences between age groups. Those aged under 6 years (I1) have higher $\delta^{15} \mathrm{~N}$ values, most probably because they had not yet been weaned, while individuals aged 6 to 14 years (I2) had lower $\delta^{13} \mathrm{C}$ and $\delta^{15} \mathrm{~N}$ values than adults ( $c f$. Hull \& O'Connell 2011; Burt 2013; Richards et al. 2002).

Somewhat surprisingly, by contrast, no significant age-dependent differences are evident at Polhill (Fig. 3). Even among children (n=9) in the sample, I (0 to 14 years) and I2 individuals' isotope values did not in general differ significantly from adults $(n=32)$ and therefore were not obviously differentiated through diet. Two I2 children had higher $\delta^{15} \mathrm{~N}$ than the others: POL72 (11.0\%) and POL100 (11.0\%). Neither had outstanding burial features so the reason for this is unclear. One possible explanation for this is if these 
individuals were at the younger end of the age-band, c. six years, they may have been weaned late. This, combined with slow turnover rates, can result in elevated $\delta^{15} \mathrm{~N}$ signals in older children (Lewis 2007). Alternatively, enriched ${ }^{15} \mathrm{~N}$ values can result from sickness or nutritional stress (Dupras et al. 2001; Katzenberg 2000), which may have been a factor here, although there is no supporting palaeopathological data for this from the site.

\subsection{Wealth}

The mean $\delta^{13} \mathrm{C}$ and $\delta^{15} \mathrm{~N}$ values for both sites grouped according to wealth determination are given in Table 6. The Melbourn sample displayed no statistically significant differences between wealth groups, or indeed between weapon $(n=6)$ and nonweapon burials, which is another potential indicator of status (Fig. 4). Rather, mean $\delta^{13} \mathrm{C}$ and $\delta^{15} \mathrm{~N}$ values for all groups were tightly clustered. The archaeological social stratification hinted at in the grave-goods was not obviously borne out through food consumption at Melbourn.

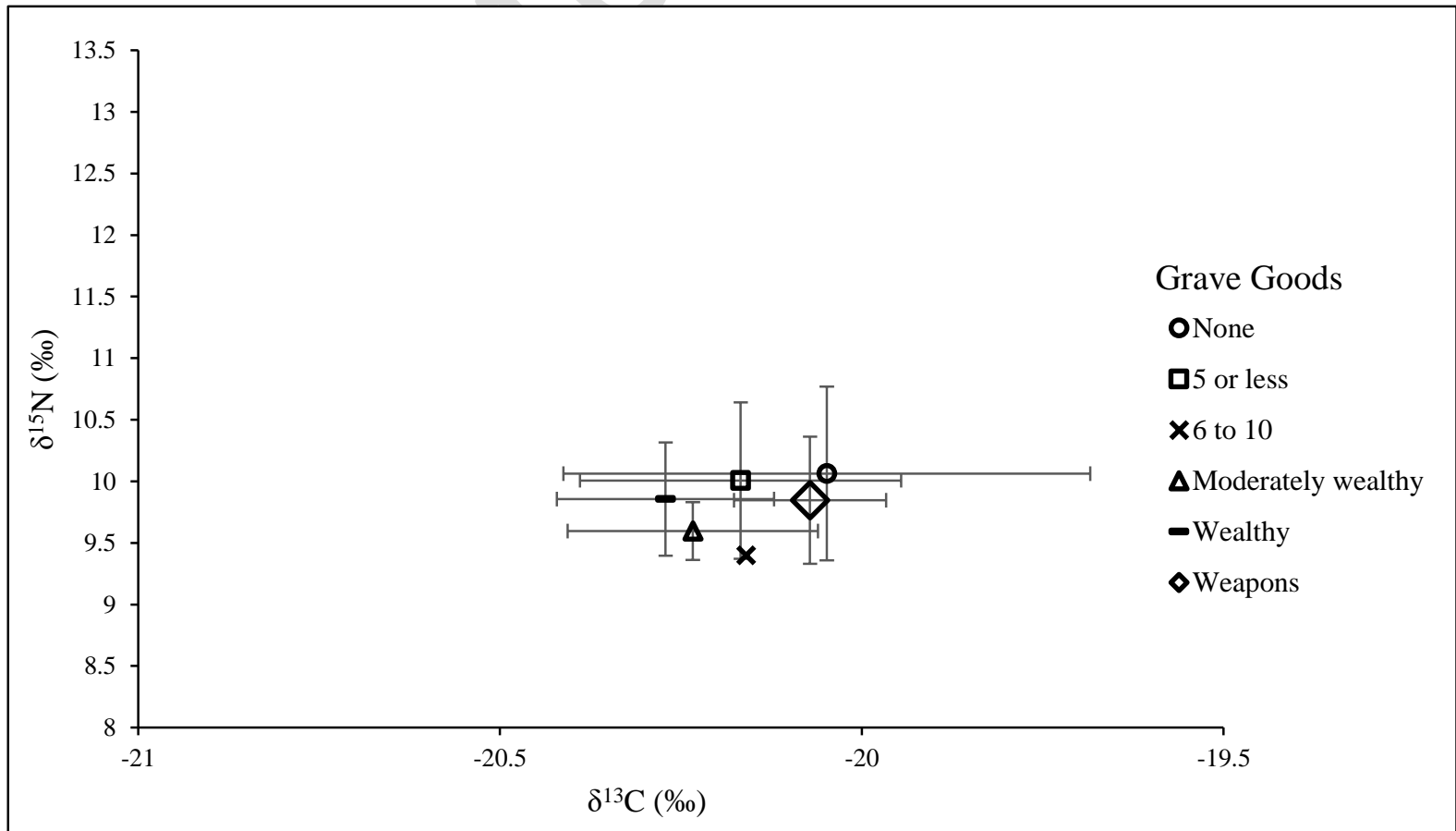

Figure 4. Mean values and standard deviations for grave-good wealth groups at Melbourn. 


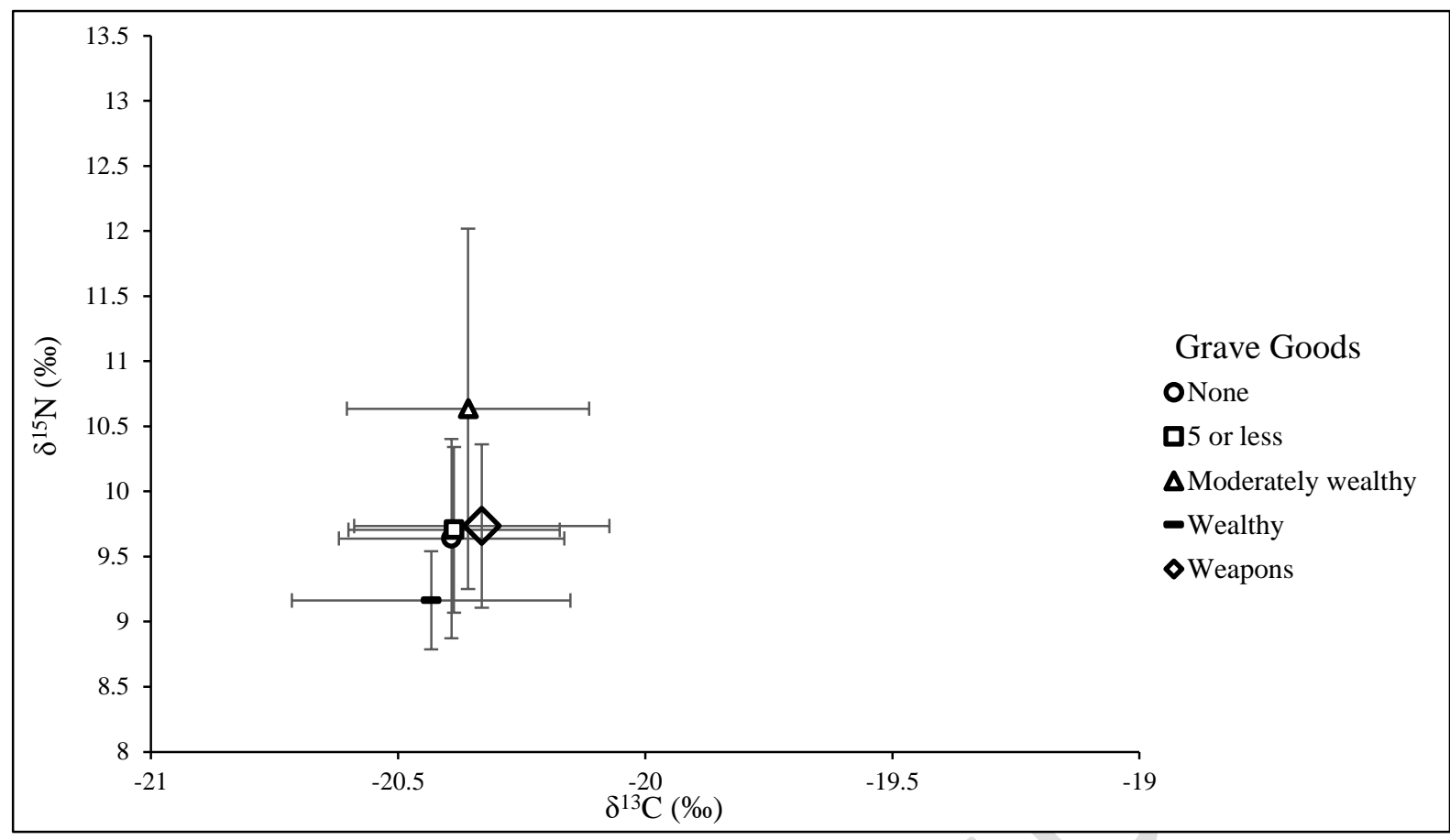

Figure 5. Mean values and standard deviations for grave-good wealth groups at Polhill.

Wealth-based differences are more readily discernible at Polhill (Figure 5). Differences were most pronounced in the $\delta^{15} \mathrm{~N}$ values, with 'wealthy' $(\mathrm{n}=6)$ and 'moderately wealthy' $(n=6)$ groups were noticeably apart from the others. In many past societies it has been shown that archaeological differences in wealth can predict palaeodiet, due to the dietary differences that existed between people of high and low social rank (Knipper et al. 2015). At Polhill, the 'wealthy' and 'moderately wealthy' means lie at either extreme of the overall $\delta^{15} \mathrm{~N}$ range, 9.2\%o and $10.6 \%$ respectively. The two wealthy and 'none/basic (5 or less)' groups had statistically significant differences in $\delta^{15} \mathrm{~N}$ values $(\mathrm{F}(2,62)=6.311, \mathrm{p}<0.05(=0.003))$. Posthoc Bonferroni tests showed that the 'none/basic' and 'moderately wealthy' groups $(\mathrm{p}=0.012)$ differed as did the 'moderately wealthy' and 'wealthy' groups $(\mathrm{p}=0.003)$. Although differences between the groups exist, their interpretation is somewhat difficult, as there is no consistent correlation between the wealth and dietary factors. Weapon $(n=10)$ and nonweapon burials were found to be indistinct. 


\subsection{Burial Feature}

Table 6 shows the mean values for $\delta^{13} \mathrm{C}$ and $\delta^{15} \mathrm{~N}$ at both sites according to other notable burial features. At each cemetery, only one distinct feature was present, each of a different form and not directly comparable. At Melbourn, this factor was the variety of body arrangements present. Whether this was linked to social organisation or not, the isotopic data shows that this differentiation in death does not correspond to dietary distinctions in life. At Polhill, this factor consisted of different grave forms, which provided a strong indicator that certain members of society were distinguished from others. Although a cursory inspection of the data suggests there were relatively consistent isotopic differences between grave forms, especially in terms of $\delta^{15} \mathrm{~N}$. Those in multiple burials $(n=26)$ had the lowest ${ }^{15} \mathrm{~N}$ values of any grave form; suggesting a diet lacking in meat, and interestingly these burials were also associated with unspectacular grave-goods. However, the differences were not statistically significant due to the variability of the results from single burials.

\section{Discussion}

We can infer from our data that there were no sex-based dietary differences in either the Melbourn or Polhill cemetery populations. Gendered roles for individuals can be inferred from the funerary record (cf. Williams 2011) which could provoke speculation as to whether there were dietary differences between sexes too, but we have not found the data to substantiate this. The diets of children appear to have been differentiated from adult groups at Melbourn. At Polhill, wealth seems to have been a determining factor in diet, although it is somewhat strange that it was the 'moderately wealthy' and not the wealthiest group that had the highest $\delta^{15} \mathrm{~N}$ values and hence the best evidence for the consumption of high-status food 
such as meat. At the Anglo-Saxon cemetery of Berinsfield in Oxfordshire, the wealthiest individuals similarly had the lowest $\delta^{15} \mathrm{~N}$ values but there, paradoxically, the poorest individuals who had the highest $\delta^{15} \mathrm{~N}$ (Privat et al. 2002). The situation at Polhill is different. It is unclear why those with middling wealth were set apart and consumed more animal protein than others. This incongruity of dietary organisation and social stratification between populations is not unique to England at this time. In early medieval (seventh to ninth centuries) southern Bavaria, the cemeteries of Großmehring and Etting produced isotopic evidence for individuals consuming more animal protein which correlated with the archaeological evidence for high-status (i.e. burial in distinct, separate, circular enclosures) (Czermak et al. 2006). In contrast, the isotopic and archaeological evidence from Kelheim cemetery was indicative of a 'less socially stratified' community (Czermak et al. 2006). In a follow-on study with the addition of two more cemeteries (Enkering and Unterigling), Strott et al. (2008) also were unable to identify dietary stratification patterns common to all populations. It is clear that the relationships between wealth, status and diet in the past were highly nuanced, and as the work reported here demonstrates, it is perhaps naïve to expect a simple correlation between the archaeological and palaeodietary factors. Indeed, this is also true of evidence for social stratification throughout the archaeological record. At both cemeteries, those individuals buried with a relatively large number of grave-goods were not necessarily found in a distinct grave form such as a coffin or mound. Similarly, there was no clear pattern in body arrangement. Discrepancies between archaeological indicators such as these remind us that a relationship between status and apparent "wealth" should not always be assumed and is likewise complex.

In this analysis, the influence of religion on diet forms only a minor component but is nonetheless important to consider due to the Conversion-period context of the cemeteries. There was no evidence for elevated fish consumption or any other distinct signal which cross- 
cuts all factions of society regardless of age, sex, or status at either site studied. Either of these signals may have suggested a religious influence of dietary patterns. It should be noted that despite the burials at Melbourn and Polhill dating to the Conversion period, there is no explicit ecclesiastical association at either site - whether by known historical association, as is the case at Westfield Farm and its proximity to Ely monastery (Lucy et al. 2009) or from grave-goods; e.g. the cross pendant at Westfield Farm (Lucy et al. 2009) or the silver spoons inscribed with the names Saul and Paul, both Christian saints, from Sutton Hoo (Carver 1998).

Mean values for Melbourn and Polhill, alongside other earlier and contemporary Anglo-Saxon cemetery populations, are displayed in Figure 6. Published faunal data from regions as close to the sites possible provide information on dietary sources and a baseline for the human data (Figure 7; Table 7; see also Figure 1 for locations).

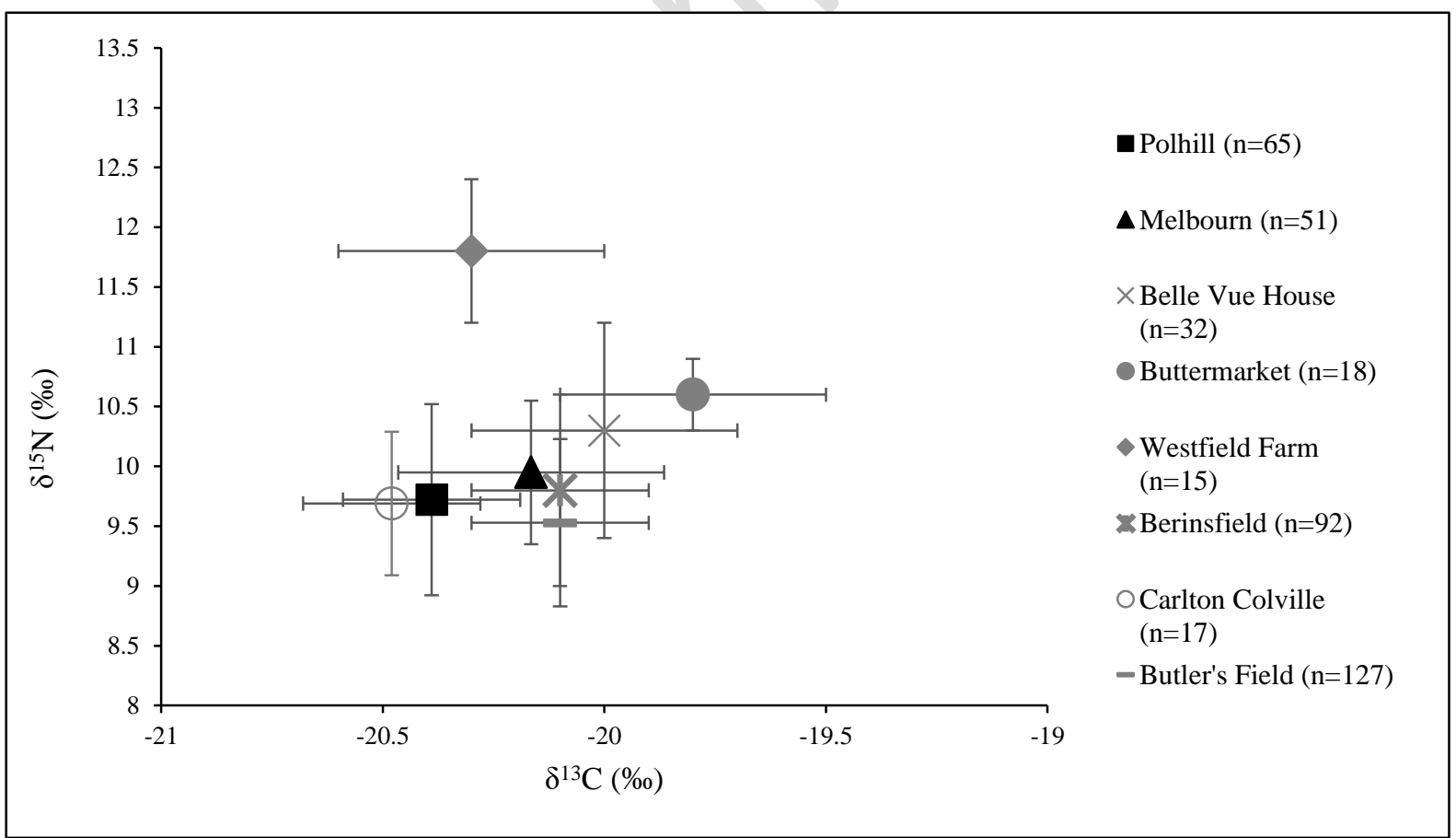

Figure 6. Mean $\delta^{13} \mathrm{C}$ and $\delta^{15} \mathrm{~N}$ with standard deviations for various Anglo-Saxon cemetery sites (Data from: Müldner \& Richards 2007; Scull 2009; Lucy et al. 2009; Privat et al. 2002; O’Connell \& Lawler 2009; O’Connell \& Wilson 2008). 


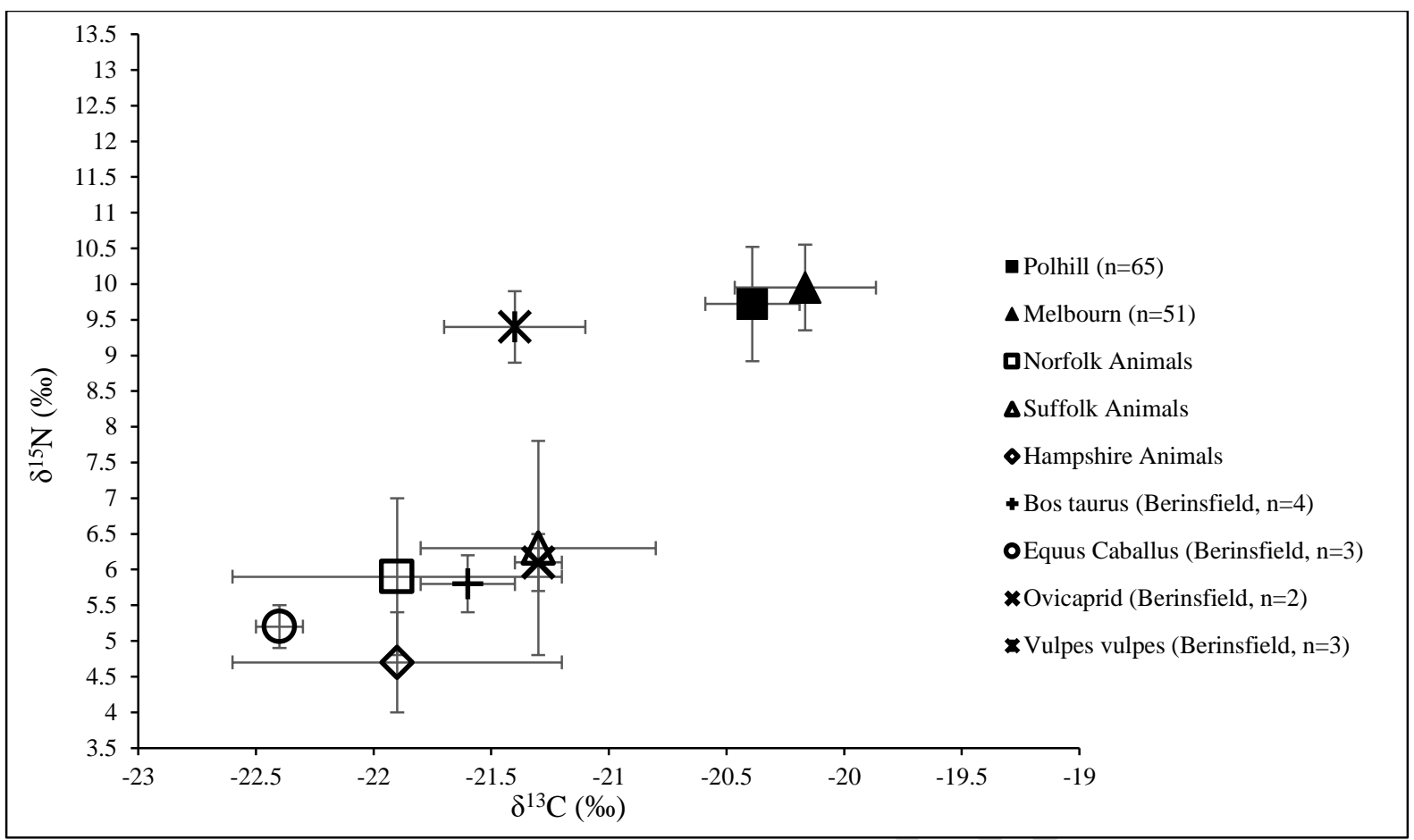

Figure 7. Mean $\delta^{13} \mathrm{C}$ and $\delta^{15} \mathrm{~N}$ values with standard deviations for faunal data and study sites (Data from: Privat et al. 2002; Hull \& O’Connell 2011).

\begin{tabular}{|c|c|c|c|}
\hline Region/Species & $\begin{array}{c}\text { Animal } \delta^{13} \mathrm{C} \% \\
( \pm \mathrm{sd})\end{array}$ & $\begin{array}{c}\text { Animal } \delta^{15} \mathbf{N} \% \\
( \pm \mathrm{sd})\end{array}$ & Reference \\
\hline Norfolk & $-21.9 \pm 0.7$ & $5.9 \pm 1.1$ & Lucy et al. 2009 \\
\hline Suffolk & $-21.3 \pm 0.5$ & $6.3 \pm 1.5$ & Lucy et al. 2009 \\
\hline Hampshire & $-21.9 \pm 0.7$ & $4.7 \pm 0.7$ & Hull \& O'Connell 2011 \\
\hline Berinsfield Bos taurus & $-21.6 \pm 0.2$ & $5.8 \pm 0.4$ & Privat et al. 2002 \\
\hline $\begin{array}{c}\text { Berinsfield Equus } \\
\text { caballus }\end{array}$ & $-22.4 \pm 0.1$ & $5.2 \pm 0.3$ & Privat et al. 2002 \\
\hline Berinsfield Ovicaprid & $-21.3 \pm 0.1$ & $6.1 \pm 0.4$ & Privat et al. 2002 \\
\hline Berinsfield Vulpes vulpes & $-21.4 \pm 0.3$ & $9.4 \pm 0.5$ & Privat et al. 2002 \\
\hline
\end{tabular}

Table 7: Baseline faunal isotope data (mean \pm standard deviation).

The fauna from Berinsfield provide the best -- and only -- appropriate comparison, being located between Melbourn and Polhill. Carlton Colville is exceptional in its coastal regional location and he animal assemblages there were very small, hampering statistical analysis. The 
$\delta^{13} \mathrm{C}$ values of the faunal data ranged from $-22.4 \%$ to $-21.3 \%$ while $\delta^{15} \mathrm{~N}$ values ranged from 4.7\%o to 9.4\% . As would be expected, the human $\delta^{15} \mathrm{~N}$ values from Melbourn and Polhill are enriched by $\sim 4-6 \%$ relative to the Berinsfield herbivore species, indicating the source of much of the dietary protein. Regional differences between Anglo-Saxon cemeteries is slight; for Melbourn and Polhill, it is likely this is the result of dietary choice as opposed to the environment, which is similar at the two locations. Within- and between-group variability is also limited. Individually, each site's ranges were narrow, with $\delta^{13} \mathrm{C}$ typically being narrower than $\delta^{15}$ N. Hull and O'Connell (2011) identified a similar pattern in their twenty-two-site review, as did Mays and Beavan (2012). The existing evidence points to a remarkably similar diet for numerous Anglo-Saxon communities, including most of those earlier than and contemporary to Melbourn and Polhill.

Individuals from Westfield Farm (later seventh century), Buttermarket (seventh century) and Belle Vue House (late seventh to early eighth centuries) display elevated $\delta^{15} \mathrm{~N}$ values relative to their contemporaries at Melbourn and Polhill, or any other site. This is particularly striking given that Westfield Farm and Melbourn are only separated by $38 \mathrm{~km}$, both located within Cambridgeshire. The isotope values at Westfield may have resulted from the cemetery's proximity to the River Ouse and its location within the Fens, leading to the greater consumption of freshwater fish (Lucy et al. 2009). Melbourn's more inland location may have rendered the population there incapable of exploiting these same resources. Alternatively, and more tentatively, diet at Westfield Farm may have been influenced by Christian practices of fasting and the requirement to eat certain foods during certain times (Lucy et al. 2009). If so, it is interesting that religion influences one community and not another, in the same region. Melbourn has almost identical mean values to the earlier site of Berinsfield (fifth to sixth century) and is also very similar to the broadly contemporary Butler's Field (late fifth to late seventh century). This suggests remarkable dietary continuity 
over time in terms of protein sources. Polhill and Carlton Colville (mid-late seventh century) also display notable similarities. In summary, Anglo-Saxon diets were broadly similar across different communities separated by both time and space. However, the albeit limited variations that have occasionally been detected between contemporary sites in different geographical regions, stresses the need for ongoing careful consideration of subtle differences in diet.

Disregarding the slight distinctions seen in the wealthier Polhill groups and the childadult division at Melbourn, the presence of otherwise negligible differences between most social groups raises the question as to whether they are meaningful at all. It is reasonable to suggest that the majority of differences may instead simply reflect biological variation between individuals. The basic premise that dietary differences are always reflected in the isotopic composition of collagen should also be questioned. As we have demonstrated, it cannot be assumed that differences in diet will be substantial enough to leave a signature, despite archaeological and historical indications that such differences may be expected. If social differentiation through food consumption was not highly prioritised or stringently upheld, we would expect the relatively narrow isotopic ranges that we found. No one group differs isotopically from another by a substantial degree. At Melbourn and Polhill, outward displays of social distinction, those made through the number and quality of grave-goods and grave form, took precedence over any which could be made through the daily intake of food. This serves as a stark reminder that archaeologically-visible statements made about an individual in death may in fact distort reality in life, or represent a different facet of it. Hull and O'Connell (2011) point out that elevated $\delta^{15} \mathrm{~N}$ values may result from the consumption of 'poor' animal sources, such as domestic birds like chicken. If this was the case, poorer individuals should exhibit higher $\delta^{15} \mathrm{~N}$ values than wealthier groups (Hull \& O'Connell 2011). This pattern was absent at Melbourn and Polhill. If stratification was not as absolute as 
archaeological evidence suggests, one can begin to question how accurate notions of food more likely to be consumed by poorer individuals, like domestic birds, and those by wealthier groups, like meat from cattle (Crawford 2009; Hull \& O’Connell 2011), in everyday AngloSaxon diet really are.

Another limitation of the current study is the potential noise introduced by different skeletal elements sampled for analysis, given the differing turnover rates of bone tissue. A productive next step in investigating diet in the Conversion period would be to study stable isotopes in tandem with other proxy indicators of diet such as the prevalence of developmental disease, tooth wear, archaeobotany, zooarchaeology, palaeoecology, and historic records. This may reveal information about rural subsistence that no one proxy can reveal on its own.

\section{Conclusions}

Our comparative study analysed samples from 116 individuals from the cemeteries at Melbourn and Polhill, mainly used during the seventh century. The isotopic data revealed that diets in both communities were noticeably similar, indicative of diets based on temperate, terrestrial $\mathrm{C}_{3}$ plants, with the regular consumption of animal protein and possibly some freshwater resources. Closer examination reveals only negligible differences between certain social groups.

In their wider multi-regional setting, Anglo-Saxon populations display overwhelmingly similar isotopic ranges, even with those that are chronologically earlier and later. This perceived uniformity reveals that food was an element of daily life which was relatively immune to drastic change and which remained largely consistent, against a 
backdrop of social reform. Even the extreme religious changes of the time do not appear to have influenced diet, at least not in any clear, isotopically-visible way.

Much like the process of social stratification, the conversion to Christianity and any obligation it may have stipulated was not simple and rigidly set from the beginning. Dietary differences may still have been enforced, for example whether certain groups had certain meat cuts or obtained protein from meat or animal by-products, but the evidence here suggests no one social group consumed significantly more quantities of animal protein or terrestrial plants than any other. This still speaks of social homogeneity of sorts. The often stated increasingly hierarchical nature of society from the sixth and seventh centuries can be challenged, at least in this aspect of daily life. This study highlights the underlying complexities of the process of social stratification, which is so commonly considered one of the main characteristics of the sixth to seventh centuries. From the isotopic data, it appears that individual communities intentionally chose their own mode of social organisation (or lack thereof), at least through diet.

\section{Acknowledgements}

We are grateful to Royal College of Surgeons for granting access the skeletal remains from Polhill and Corinne Duhig (University of Cambridge) for all her help in obtaining these permissions. Also to Quentin Carroll, and those in the Cambridgeshire County Council Historic Environment Team, for granting access to the Melbourn collection. We thank Tamsin O'Connell for her guidance during the course of the work as well as subsequent discussions and comments. We also thank Catherine Kneale (McDonald Institute for Archaeological Research) for her valuable assistance with the isotopic analysis and also to James Rolfe (Godwin Laboratory) for help. This work was completed during postgraduate 
study made possible through funding by the Cambridge Arts and Humanities Research

Council Doctoral Training Partnership Award (2014-15).

\section{Bibliography}

Ambrose, S. H. 1990. 'Preparation and Characterization of Bone and Tooth Collagen for Isotopic Analysis.' Journal of Archaeological Science. Vol. 17. P.431-451.

Banham, D. 2004. Food and Drink in Anglo-Saxon England. Stroud: Tempus.

Barrett, J. H. \& Richards, M. P. 2004. 'Identity, Gender, Religion and Economy: new isotope and radiocarbon evidence from marine resource intensification in early historic Orkney, Scotland, UK.' European Journal of Archaeology. Vol. 7. P. 249-271.

Blair, J. 2005. The church in Anglo-Saxon society. Oxford: Oxford University Press.

Bocherens, H. \& Drucker, D. G. 2003. 'Trophic level isotopic enrichment of carbon and nitrogen in bone collagen: case studies from recent and ancient terrestrial ecosystems.' International Journal of Osteoarchaeology. Vol. 13. P. 46-53.

Brown, T. \& Brown, K. 2011. Bimolecular Archaeology: an introduction. Chichester: WileyBlackwell.

Burt, N. M. 2013. 'Stable Isotope Ratio Analysis of Breastfeeding and Weaning Practices of Children from Medieval Fishergate House York, UK.' American Journal of Physical Anthropology. Vol. 152. P. 407-416.

Carver, M. 1998. Sutton Hoo: burial ground of kings? London: British Museum Press.

Chisholm, B. S., Nelson, D. E. \& Schwarcz, H. P. 1982. 'Stable-Carbon Isotope Ratios as a Measure of Marine Versus Terrestrial Protein in Ancient Diets.' Science. Vol. 216. P. 1131-1132.

Crawford, S. 2009. Daily Life in Anglo-Saxon England. Oxford: Greenwood World Publishing.

Czermak, A., Ledderose, A., Strott, N., Meier, T. \& Grupe, G. 2006. 'Social Structures and Social Relations - An Archaeological and Anthropological Examination of three Early Medieval Separate burial sites in Bavaria.' Anthropologischer Anzeiger. Vol. 64. P. 297-310.

DeNiro, M. J. 1985. 'Postmortem preservation and alteration of in vivo bone collagen isotope ratios in relation to palaeodietary reconstruction.' Nature. Vol. 317. P. 806-809.

DeNiro, M. J. \& Epstein, S. 1981. 'Influence of diet on the distribution of nitrogen isotopes in animals.' Geochimica et Cosmochimica Acta. Vol. 45. P. 341-351.

Dufour, E., Bocherens, H. \& Mariotti, A. 1999. 'Palaeodietary Implications of Isotopic Variability in Eurasian Lacustrine Fish.' Journal of Archaeological Science. Vol. 26. P. 617-627. 
Duncan, H., Duhig, C. \& Phillips, M. 2003. 'A Late Migration/Final Phase cemetery at Water Lane, Melbourn.' Proceedings of the Cambridge Antiquarian Society. Issue 92. P. 57134.

Dupras, T. L., Schwarcz, H. P. \& Fairgrieve, S. I. 2001. 'Infant Feeding and Weaning Practices in Roman Egypt.' American Journal of Physical Anthropology. Vol. 115. P. 204-212.

Ervynck, A. 1997. 'Following the Rule? Fish and Meat consumption in Monastic Communities in Flanders (Belgium)', in: De Boe, G. and Verhaeghe, F. (eds.) Environment and Subsistence in Medieval Europe, Papers of the 'Medieval Europe Brugge 1997 Conference' Volume 9. Zellik: Instituut voor het Archeologisch Patrimonium. P.67-81.

Frantzen, A. J. 2014. Food, Eating and Identity in Early Medieval England. Woodbridge: Boydell Press.

Gardiner, M. 2011. 'Late Saxon Settlements,' in: Hamerow, H., Hinton, D. A. and Crawford, S. (eds.) The Oxford Handbook of Anglo-Saxon Archaeology. Oxford: Oxford University Press. P. 198-217.

Geake, H. 1997. The Use of Grave-Goods in Conversion-Period England, c. 600- c. 850. Oxford: John and Erica Hedges.

Hedges, R. E. M., Clement, J. G., David, C., Thomas, L. \& O’Connell, T. 2007. 'Collagen turnover in the adult femoral mid-shaft: Modelled from anthropogenic radiocarbon tracer measurements.' American Journal of Physical Anthropology. Vol. 133. P. 808816.

Hedges, R. E. M. \& Reynard, L. M. 2007. 'Nitrogen isotopes and the trophic level of humans in archaeology.' Journal of Archaeological Science. Vol. 34. P. 1240-1251.

Higham, N. J., \& Ryan. M. J. 2013. The Anglo-Saxon World. New Haven: Yale University Press.

Hoggett, R. 2010. The Archaeology of the East Anglian Conversion. Woodbridge: Boydell.

Hull, B. D. \& O' Connell, T. C. 2011. 'Diet: Recent Evidence from Analytical Chemical Techniques', in: Hamerow, H., Hinton, D. A. and Crawford, S. (eds.) The Oxford Handbook of Anglo-Saxon Archaeology. Oxford: Oxford University Press. P. 667687.

Katzenberg, M. A. 2000. 'Stable Isotope Analysis: A Tool for Studying Past Diet, Demography, and Life History,' in: Katzenberg, M. A. and Saunders, S. R. (eds.) Biological Anthropology of the Human Skeleton. New York; Chichester: Wiley-Liss. P. 305-327.

Keaveney, E., Reimer, P. J. \& Foy, R. H. 2015. 'Young, Old, and Weathered Carbon- Part 2: Using Radiocarbon and Stable Isotopes to Identify Terrestrial Carbon Support of the Food Web in an Alkaline, Humic Lake.' Radiocarbon Journal. Vol. 57. P. 425-438.

Knipper, C., Held, P., Fecher, M., Nicklisch, N., Meyer, C., Schreiber, H., Zich, B., MetznerNebelsick, C., Hubensack, V., Hansen, L., Nieveler, E., and Alt, K. W. 2015. 'Superior in Life-Superior in Death: Dietary Distinction of Central European Prehistoric and Medieval Elites.' Current Anthropology. Vol. 56. P. 579-589. 
Lee-Thorp, J. A. 2008. 'On Isotopes and Old Bones.' Archaeometry. Vol. 50. P. 925-950.

Lewis, M. E. 2007. The Bioarchaeology of Children: Perspectives from Biological and Forensic Anthropology. Cambridge: Cambridge University Press.

Lucy, S. 2000. The Anglo-Saxon Way of Death. Stroud: Sutton.

Lucy, S., Newman, R., Dodwell, N., Hills, C., Dekker, M., O’ Connell, T., Riddler, I. \& Walton Rogers, P. 2009. 'The Burial of A Princess? The Later Seventh-Century Cemetery at Westfield Farm, Ely.' The Antiquaries Journal. Vol. 89. P. 81-141.

Malainey, M. E. 2011. A Consumer's Guide to Archaeological Science: Analytical Techniques. New York: Springer.

Mays, S. A. 1997. 'Carbon Stable Isotopes Ratios in Medieval and Later Human Skeletons From Northern England.' Journal of Archaeological Science. Vol. 24. P. 561-567.

Mays, S. \& Beavan, N. 2012. 'An investigation of diet in early Anglo-Saxon England using carbon and nitrogen stable isotope analysis of human bone collagen.' Journal of Archaeological Science. Vol. 39. P. 867-874.

Müldner, G. \& Richards, M. P. 2005. 'Fast or feast: reconstructing diet in later medieval England by stable isotope analysis.' Journal of Archaeological Science. Vol. 32. P. $39-48$.

Müldner, G. \& Richards, M. P. 2007. 'Stable Isotope Evidence for 1500 Years of Human Diet at the City of York, UK.' American Journal of Physical Anthropology. Vol. 133. P. 682-697.

O'Connell, T. \& Lawler, A. 2009. 'Stable isotope analysis of human and faunal remains,' in: Lucy, S., Tipper, J. and Dickens, A. The Anglo-Saxon Settlement and Cemetery at Bloodmoor Hill, Carlton Colville, Suffolk. Cambridge: Cambridge Archaeological Unit. P. 317-321.

O'Connell, T. C. \& Wilson, E. J. 2008. 'Stable isotope analysis of human remains from the Anglo-Saxon cemetery at Butler's Field, Lechdale, Gloucestershire: dietary and social implications,' in Boyle, A., Jennings, D., Miles, D. and Palmer, S. (eds.) The AngloSaxon Cemetery at Butler's Field, Lechdale, Gloucestershire, Volume 2. Oxford: Oxford Archaeological Unit.

Philp, B. 1973. Excavations in West Kent 1960-1970: The Discovery and Excavation of Prehistoric, Roman, Saxon and Medieval Sites, mainly in the Bromley area and in the Darant Valley. Kent: Kent Archaeological Rescue Unit.

Polet, C. \& Katzenberg, M. A. 2003. 'Reconstruction of the diet in a mediaeval monastic community from the coast of Belgium.' Journal of Archaeological Science. Vol. 30. P. 525-533.

Privat, K. L., O' Connell, T. C. \& Richards, M. P. 2002. 'Stable isotope analysis of human and faunal remains from the Anglo-Saxon cemetery at Berinsfield, Oxfordshire: dietary and social implications.' Journal of Archaeological Science. Vol. 29. P. 779790 .

Richards, M. P. \& Hedges, R. E. M. 1999. 'Stable Isotope Evidence for Similarities in the Types of Marine Foods Used by Late Mesolithic Humans at Sites Along the Atlantic Coast of Europe.' Journal of Archaeological Science. Vol. 26. P. 717-722. 
Richards, M. P., Mays, S. \& Fuller, B. T. 2002. 'Stable Carbon and Nitrogen Isotope Values of Bone and Teeth Reflect Weaning Age at the Medieval Wharram Percy Site, Yorkshire, UK.' American Journal of Physical Anthropology. Vol. 119. P. 205-210.

Schoeninger, M. J. 1989. 'Reconstructing prehistoric human diet,' in: Price, D. T. (ed.) The Chemistry of Prehistoric Human Bone. Cambridge: Cambridge University Press. P. 38-67

Schoeninger, M. J. \& DeNiro, M. J. 1984. 'Nitrogen and carbon isotopic composition of bone collagen from marine and terrestrial animals.' Geochimica et Cosmochimica Acta. Vol. 48. P. 625-639.

Scull, C. 2009. Early medieval (late $5^{\text {th }}$-early $8^{\text {th }}$ centuries AD) cemeteries at Boss Hall and Buttermarket, Ipswich, Suffolk. Leeds: Society for Medieval Archaeology.

Simon, G. A. 2009. Commentary for Benedictine Oblates: On the Rule of St. Benedict. Eugene: Wipf and Stock Publishers.

Simoons, F. J. 1994. Eat Not This Flesh: Food Avoidances from Prehistory to Present. $2^{\text {nd }}$ ed. Madison: The University of Wisconsin Press.

Strott, N., Czermak, A. \& Grupe, G. 2008. 'Are biological correlates to social stratification depicted in skeletal finds? Investigation of early medieval separate burial grounds in Bavaria.' Documenta Archaeobiologiae. Vol. 5. P. 68-86.

Ulmschneider, K. 2011. 'Settlement Hierarchy,' in: Hamerow, H., Hinton, D. A. and Crawford, S. (eds.) The Oxford Handbook of Anglo-Saxon Archaeology. Oxford: Oxford University Press. P. 156-171.

Welch, M. 2011. 'The Mid Saxon 'Final Phase',' in: Hamerow, H., Hinton, D. A. and Crawford, S. (eds.) The Oxford Handbook of Anglo-Saxon Archaeology. Oxford: Oxford University Press. P. 266-287.

Williams, H. 2011. 'Mortuary Practices in Early Anglo-Saxon England,' in: Hamerow, H., Hinton, D. A. and Crawford, S. (eds.) The Oxford Handbook of Anglo-Saxon Archaeology. Oxford: Oxford University Press. P. 238-265.

Wilson, D. 1956. 'The Initial Excavation of an Anglo-Saxon Cemetery at Melbourn, Cambridgeshire.' Proceedings of the Cambridge Antiquarian Society. Issue 46. P. 2941. 